\title{
On 'orbital' and 'spin' angular momentum of light in classical and quantum theories - a general framework
}

\author{
Arvind* \\ Department of Physical Sciences, Indian Institute of Science Education \& Research (IISER) Mohali, \\ Sector 81 SAS Nagar, Manauli PO 140306 Punjab India. \\ S. Chaturved \\ Department of Physics, Indian Institute of Science Education 6 Research (IISER) Bhopal, \\ Bhopal Bypass Road, Bhauri, Bhopal 462066 India \\ N. Mukundaf \\ INSA Distinguished Professor, Indian Academy of Sciences, \\ C V Raman Avenue, Sadashivanagar, Bangalore 560080 India
}

\begin{abstract}
We develop a general framework to analyze the two important and much discussed questions concerning (a) 'orbital' and 'spin' angular momentum carried by light and (b) the paraxial approximation of the free Maxwell system both in the classical as well as quantum domains. After formulating the classical free Maxwell system in the transverse gauge in terms of complex analytical signals we derive expressions for the constants of motion associated with its Poincaré symmetry. In particular, we show that the constant of motion corresponding to the total angular momentum $\mathbf{J}$ naturally splits into an 'orbital' part $\mathbf{L}$ and a 'spin' part $\mathbf{S}$ each of which is a constant of motion in its own right. A noteworthy feature of the formulation so developed is the emergence of a complex Hilbert space $\mathcal{M}$ associated with the free Maxwell system which in turn provides a natural link between the descriptions of radiation fields at the classical and quantum levels. We then proceed to discuss quantization of the free Maxwell system and construct the operators generating the Poincaré group in the quantum context and analyze their algebraic properties and find that while the quantum counterparts $\hat{\mathbf{L}}$ and $\hat{\mathbf{S}}$ of $\mathbf{L}$ and $\mathbf{S}$ go over into bona fide observables, they fail to satisfy the angular momentum algebra precluding the possibility of their interpretation as 'orbital' and 'spin' operators at the classical level. On the other hand $\hat{\mathbf{J}}=\hat{\mathbf{L}}+\hat{\mathbf{S}}$ does satisfy the angular momentum algebra and together with $\hat{\mathbf{S}}$ generates the group $E(3)$. We then present an analysis of single photon states, paraxial quantization both in the scalar as well as vector cases, single photon states in the paraxial regime. All along a close connection is maintained with the Hilbert space $\mathcal{M}$ that arises in the classical context thereby providing a bridge between classical and quantum descriptions of radiation fields. The present approach provides strong motivation for making the paraxial approximation after, and not before, field quantization has been carried out.
\end{abstract}

\section{INTRODUCTION}

There has been much interest recently in the theoretical understanding and experimental exploitation of the angular momentum properties of light in both classical and quantum domains [1]. It has been shown that the total angular momentum of the (free) electromagnetic field, a well-defined expression which is conserved on account of Poincaré invariance, can be meaningfully separated into 'orbital' and 'spin' parts, each of which is conserved by itself 2]. (For brevity the former will be called the OAM - orbital angular momentum - of light). Through experiments it has been suggested that specific effects attributable to the OAM of light can be identified. Schemes to filter OAM states have been proposed [3, 4] and proposals for beam splitters for the OAM states aimed at achieving quantum information processing have also been

\footnotetext{
*Electronic address: arvind@iisermohali.ac.in

$\dagger$ Electronic address: subhash@iiserbhopal.ac.in

${ }^{\ddagger}$ Electronic address: nmukunda@gmail.com
}

developed [5]. There have also been several discussions on the cloning of OAM states [6] and network-based OAM [7]. The quantum states with OAM have been proposed to be useful for implementation of quantum gates [8] and for universal quantum computation [9, 10]. Quantum key distribution schemes based on OAM have also been proposed 11, 12 and the possibility of creating entangled photons has also been discussed [13]. Entanglement of OAM states has been demonstrated experimentally [14]. Experiments have also been performed on implementing quantum random walks [15] and quantum cryptographic schemes using quantum states of light with OAM 16]. Squeezing of states with OAM has been experimentally demonstrated [17] and various correlation experiments have also been carried out [18, 19]. Single photon in modes with OAM have been prepared 20] and Laguerre-Gaussian laser modes with OAM have also been generated [21].

The properties of OAM of light for the practically important paraxial beams are important as laser beam modes are of this nature. Another aspect is to see whether anything new can be learnt about the quantum mechanics of single photons, both in terms of possible 
physical observables and in the properties of particular quantum states. A comprehensive analysis is contained in the work of Calvo et. al. 22], in which a systematic approximation scheme for paraxial beams has been proposed. In addition the procedure to be adopted for field quantization to arrive at a physically reasonable concept of 'paraxial photons' has been addressed by Deutsch et. al. 23]. Here it may be recalled that single photons with fixed helicity are described by specific mass zero unitary irreducible representations (UIR's) of the Poincaré group not including the parity operation 24]. A photon with right (or left) circular polarization remains so under all proper rotations as well as under all pure Lorentz transformations. Examining these representations in detail, Newton and Wigner 25] found in a classic analysis that no position operator with reasonable physical properties can be defined for photons. This result can be expected to have important consequences for the existence of OAM as a meaningful and measurable property for single photons, and therefore also for the quantized field.

The purpose of the present work is to analyze carefully the properties and interconnections of the different concepts mentioned above - OAM for classical light; the specific algebraic properties needed for a vectorial dynamical variable to be an 'angular momentum'; related properties at the level of single photon states; and the details of the paraxial limit for light in relation to quantization. The paraxial regime for light beams is by its very nature defined in a qualitative and approximate way. Therefore the relationship between it and the quantization process needs careful analysis. In effect, the concept of 'paraxial photons' has to be arrived at in a physically reasonable manner. In addition, there is the pervasive effect of transversality in all matters relating to electromagnetism - the fields and vector potential; the quantization process; photon wave functions, and even the definition of the paraxial regime.

The contents of the paper are organized as follows. In Section II we discuss the Poincaré covariance of the Maxwell equations in free space and derive, in the transverse radiation gauge, expressions for the associated constants of motion in terms of the real fields $\mathbf{E}(\mathbf{x}, t)$, the electric field and $\mathbf{A}(\mathbf{x}, t)$, the vector potential. With eventual passage to a description of quantized electromagnetic fields in mind we move from the real $\mathbf{A}$ and $\mathbf{E}$ fields to their complex analytic signals $\mathbf{A}^{(+)}$and $\mathbf{E}^{(+)}$ and re-express the ten constants of motion in terms of $\mathbf{A}^{(+)}(\mathbf{x}, t)$ and $\mathbf{E}^{(+)}(\mathbf{x}, t)$ and also in terms of the complex transverse $\mathbf{k}$-space amplitudes $\mathbf{v}(\mathbf{k})$ associated with $\mathbf{A}^{(+)}(\mathbf{x}, t)$. In particular, we note that the constant of motion, $\mathbf{J}$, the total angular momentum, can be regarded as a sum of 'orbital' and 'spin' parts $\mathbf{L}$ and $\mathbf{S}$ each of which is also a constant of motion in its own right. The structure of the expressions for the constants of motion obtained thus prompts us to introduce the notion of a Hilbert space $\mathcal{M}$ with $\mathbf{A}^{(+)}(\mathbf{x}, t)$ or equivalently their complex transverse $\mathbf{k}$-space amplitudes $\mathbf{v}(\mathbf{k})$ as its elements. In Section III we quantize the free space Maxwell field following the canonical route by turning the $\mathbf{k}$-space amplitudes $\mathbf{v}(\mathbf{k})$ into operators $\hat{\mathbf{a}}(\mathbf{k})$ and the fundamental Poisson brackets into canonical commutation relations. This then sets the stage for introducing the notion of Hilbert spaces $\mathcal{H}_{n}$ with $n=0,1,2, \cdots$ spanned respectively of no photon or the vacuum state, 1 photon states, 2 photon states .....and hence to the $\mathcal{H}$ associated with quantized electromagnetic field obtained by taking their direct sum. The analytic signals $\mathbf{A}^{(+)}$and $\mathbf{E}^{(+)}$now become operators $\hat{\mathbf{A}}^{(+)}$and $\mathbf{E}^{(+)}$and the classical constants of motions go over into hermitian operators on $\mathcal{H}$. With this done we compute all possible commutation relations between the components of the quantum counterparts $\hat{\mathbf{J}}, \hat{\mathbf{L}}$ and $\hat{\mathbf{S}}$ of $\mathbf{J}, \mathbf{L}$ and $\mathbf{S}$, and find that while the components of $\hat{\mathbf{J}}$ obey the angular momentum algebra, those of $\hat{\mathbf{L}}$ and $\hat{\mathbf{S}}$ fail to do so. Further while the components of $\hat{\mathbf{S}}$ commute with each other, those of $\hat{\mathbf{L}}$ and $\hat{\mathbf{S}}$ do not all commute. This circumstance calls into question decomposition of the total angular momentum operator into an 'orbital' angular momentum part $\hat{\mathbf{L}}$ and a 'spin' angular momentum part $\hat{\mathbf{S}}$ as has already been noted in the literature.

Next we define single photon states in the one photon Hilbert space $\mathcal{H}_{1}$ by associating creation-annihilation operators $\hat{a}(\mathbf{v})^{\dagger}, \hat{a}(\mathbf{v})$ with each $\mathbf{v}(\mathbf{k})$ or equivalently with each $\mathbf{A}^{(+)}(\mathbf{x}, t)$ in $\mathcal{M}$. We then detail their properties and actions of the operators corresponding to the Poincaré algebra and of the infinitesimal unitary transformations they generate on these states.

In Section IV, we turn our attention to the paraxial approximation in classical scalar and vector optics with a view to giving a meaningful definition of 'quantization in the paraxial regime'. We show, in contrast to several works in the literature, that a better procedure is to impose paraxial conditions on $\mathbf{v}(\mathbf{k})$ which appear in the definition of the one-photon states as is discussed in Section III. We conclude this Section with a discussion of Laguerre-Gaussian(L-G) modes studied extensively in scalar wave optics anticipating their extension to the vector case in the following Section. In Section V we construct the eigenfunctions of $\hat{J}_{3}$ in the single photon subspace both in the $\mathbf{k}$ space as well as in the physical space and examine their behaviors in the paraxial regime. We also extend the scalar L-G modes discussed in the previous Section to the vector case. Section VI contains a summary of this work and our concluding remarks.

\section{CLASSICAL FREE MAXWELL SYSTEM, REAL FIELDS AND ANALYTIC SIGNALS, CONSTANTS OF MOTION}

In this Section, as background, we recall basic properties of the classical Maxwell equations, and settle matters of notation. Certain features not often discussed explicitly are mentioned.

The equations of motion (EOM) for real electric and 
magnetic fields in free space are

$$
\begin{aligned}
\frac{\partial}{\partial t} \mathbf{E}(\mathbf{x}, t) & =c \nabla \wedge \mathbf{B}(\mathbf{x}, t), \\
\frac{\partial}{\partial t} \mathbf{B}(\mathbf{x}, t) & =-c \nabla \wedge \mathbf{E}(\mathbf{x}, t),
\end{aligned}
$$

supplemented by the constraints

$$
\nabla \cdot \mathbf{E}(\mathbf{x}, t)=\nabla \cdot \mathbf{B}(\mathbf{x}, t)=0
$$

which are preserved in time. In special relativistic notation $\left(x^{0}=c t ; g_{00}=-1 ; \mu, \nu=0,1,2,3\right), \mathbf{E}$ and $\mathbf{B}$ are components of a real antisymmetric second rank tensor field $F_{\mu \nu}(x)=-F_{\nu \mu}(x),(x=(c t, \mathbf{x}))$ :

$$
E_{j}(x)=F_{j 0}(x), B_{j}(x)=\frac{1}{2} \epsilon_{j l m} F_{l m}(x), j, l, m=1,2,3 .
$$

Then eqs. (2.1, 2.2) are

$$
\partial^{\mu} F_{\mu \nu}(x)=0, \quad \partial_{\lambda} F_{\mu \nu}(x)+\partial_{\mu} F_{\nu \lambda}(x)+\partial_{\nu} F_{\lambda \mu}(x)=0 .
$$

These are manifestly covariant under the action of inhomogeneous Lorentz, i.e., Poincaré, transformations. A general element $(\Lambda, a)$ of the Poincaré group $\mathcal{P}$ acts on space-time coordinates $x=\left(x^{\mu}\right)$ by

$$
x^{\mu} \rightarrow x^{\prime \mu}=\Lambda_{\nu}^{\mu} x^{\nu}+a^{\mu} .
$$

Here $\Lambda=\left(\Lambda_{\nu}^{\mu}\right) \in O(3,1)$ is a real $4 \times 4$ matrix of a homogeneous Lorentz transformation in which we allow parity but omit time reversal, and $a^{\mu}$ is a space time translation. The composition law in $\mathcal{P}$ is

$$
\left(\Lambda^{\prime}, a^{\prime}\right)(\Lambda, a)=\left(\Lambda^{\prime} \Lambda, a^{\prime}+\Lambda^{\prime} a\right),
$$

while $F_{\mu \nu}$ transforms as

$$
F_{\mu \nu}^{\prime}\left(x^{\prime}\right)=\Lambda_{\mu}^{\rho} \Lambda_{\nu}^{\sigma} F_{\rho \sigma}(x) .
$$

This action by $\mathcal{P}$ preserves eqs. (2.4).

We hereafter work in the transverse radiation gauge, to begin with using real fields $\mathbf{E}(\mathbf{x}, t), \mathbf{A}(\mathbf{x}, t)$. The EOM, constraints and definition of $\mathbf{B}$ are:

$$
\begin{aligned}
\frac{\partial}{\partial t} \mathbf{A}(\mathbf{x}, t) & =-c \mathbf{E}(\mathbf{x}, t) \\
\frac{\partial}{\partial t} \mathbf{E}(\mathbf{x}, t) & =-c \nabla^{2} \mathbf{A}(\mathbf{x}, t) \\
\nabla \cdot \mathbf{E}(\mathbf{x}, t) & =\nabla \cdot \mathbf{A}(\mathbf{x}, t)=0 \\
\mathbf{B}(\mathbf{x}, t) & =\nabla \wedge \mathbf{A}(\mathbf{x}, t) .
\end{aligned}
$$

The behaviors of $\mathbf{E}$ and $\mathbf{A}$ under spatial rotations and space-time translations are immediate. Under an infinitesimal pure Lorentz transformation we first transform $\mathbf{A}(x)$ as though it is a four-vector (with $A_{0}=0$ ), and then perform an infinitesimal gauge transformation to restore transversality:

$$
\begin{aligned}
x^{\prime 0} & \simeq x^{0}-\frac{v_{j}}{c} x_{j}, \quad x_{j}^{\prime} \simeq x_{j}-\frac{v_{j}}{c} x^{0}, \quad|\mathbf{v}|<<c: \\
\mathbf{E}^{\prime}\left(x^{\prime}\right) & \simeq \mathbf{E}(x)+\frac{1}{c} \mathbf{v} \wedge \mathbf{B}(x), \\
\mathbf{A}^{\prime}\left(x^{\prime}\right) & \simeq \mathbf{A}(x)+\frac{1}{c} \nabla\left(\frac{1}{\nabla^{2}} \mathbf{v} \cdot \mathbf{E}\right)(x) \\
& =\mathbf{A}(x)-\frac{1}{4 \pi c} \nabla \int d^{3} y \frac{\mathbf{v} \cdot \mathbf{E}(\mathbf{y}, t)}{|\mathbf{x}-\mathbf{y}|} .
\end{aligned}
$$

The basic constants of motion (COM), ten in number, associated with solutions to eqs. (2.1, 2.8) are the energymomentum four-vector $P^{\mu}$ and four-dimensional angular momentum second rank antisymmetric tensor $J_{\mu \nu}$. These are expressed in terms of an energy density $\mathcal{E}(\mathbf{x}, t)$ and a momentum density $\mathcal{P}(\mathbf{x}, t)$ :

$$
\begin{aligned}
\mathcal{E}(\mathbf{x}, t) & =\frac{1}{8 \pi}(\mathbf{E}(\mathbf{x}, t) \cdot \mathbf{E}(\mathbf{x}, t)+\mathbf{B}(\mathbf{x}, t) \cdot \mathbf{B}(\mathbf{x}, t)), \\
\mathcal{P}(\mathbf{x}, t) & =\frac{1}{4 \pi c} \mathbf{E}(\mathbf{x}, t) \wedge \mathbf{B}(\mathbf{x}, t),
\end{aligned}
$$

(the Poynting vector being $c^{2} \mathcal{P}$ ) as

$$
\begin{aligned}
P^{0} & =\int d^{3} x \mathcal{E}(\mathbf{x}, t), \quad \mathbf{P}=\int d^{3} x \mathcal{P}(\mathbf{x}, t) ; \\
\mathbf{J} & =\left(\mathrm{J}_{23}, \mathrm{~J}_{31}, \mathrm{~J}_{12}\right)=\int d^{3} x \mathbf{x} \wedge \mathcal{P}(\mathbf{x}, t) ; \\
\mathbf{K} & =\left(\mathrm{J}_{10}, \mathrm{~J}_{20}, \mathrm{~J}_{30}\right)=\frac{1}{c} \int d^{3} x \mathbf{x} \mathcal{E}(\mathbf{x}, t)-c t \mathbf{P} .
\end{aligned}
$$

In terms of $\mathbf{E}$ and $\mathbf{A}$, dropping surface terms at spatial infinity we have:

$$
\begin{aligned}
& P^{0}=\frac{1}{8 \pi} \int d^{3} x\left(\mathbf{E}(\mathbf{x}, t) \cdot \mathbf{E}(\mathbf{x}, t)-\mathbf{A}(\mathbf{x}, t) \cdot \nabla^{2} \mathbf{A}(\mathbf{x}, t)\right) \\
& \mathbf{P}=\frac{1}{4 \pi c} \int d^{3} x E_{m}(\mathbf{x}, t) \nabla A_{m}(\mathbf{x}, t) \\
& J_{j}=\frac{1}{4 \pi c} \int d^{3} x E_{m}(\mathbf{x}, t)\left(\delta_{m n}(\mathbf{x} \wedge \boldsymbol{\nabla})_{j}+\epsilon_{j m n}\right) A_{n}(\mathbf{x}, t), \\
& K_{j}(t)=\frac{1}{c} \int d^{3} x x_{j} \mathcal{E}(\mathbf{x}, t) \\
& =\frac{1}{8 \pi c} \int d^{3} x x_{j}\left(\mathbf{E}(\mathbf{x}, t) \cdot \mathbf{E}(\mathbf{x}, t)-\mathbf{A}(\mathbf{x}, t) \cdot \nabla^{2} \mathbf{A}(\mathbf{x}, t)\right) .
\end{aligned}
$$

Equations (2.8) lead to the wave equation for $\mathbf{A}(x)$. Omitting possible evanescent waves, the general solution can be written as

$$
\begin{aligned}
& \mathbf{A}(\mathbf{x}, t)=\frac{c}{2 \pi} \int_{\mathbb{R}^{3}} \frac{d^{3} k}{\sqrt{\omega}}\left(e^{i k \cdot x} \mathbf{v}(\mathbf{k})+\text { c.c }\right), \\
& \mathbf{k} \cdot \mathbf{v}(\mathbf{k})=0, k^{0}=|\mathbf{k}|=\omega / c .
\end{aligned}
$$

Thus it involves one complex transverse vector amplitude $\mathbf{v}(\mathbf{k})$ on wave vector space. The real electric field is

$$
\mathbf{E}(\mathbf{x}, t)=\frac{i}{2 \pi} \int d^{3} k \sqrt{\omega}\left(e^{i k \cdot x} \mathbf{v}(\mathbf{k})-\text { c.c }\right) .
$$


In terms of $\mathbf{v}(\mathbf{k})$, the COM's and $\mathbf{K}(t)$ are:

$$
\begin{aligned}
\left(P^{0}, \mathbf{P}\right) & =\int d^{3} k\left(c k^{0}, \mathbf{k}\right) \mathbf{v}(\mathbf{k})^{*} \cdot \mathbf{v}(\mathbf{k}), \\
J_{j} & =\int d^{3} k v_{m}(\mathbf{k})^{*}\left(-i \delta_{m n}(\mathbf{k} \wedge \tilde{\nabla})_{j}-i \epsilon_{j m n}\right) v_{n}(\mathbf{k}), \\
K_{j}(t) & =\frac{i}{c} \int d^{3} k \sqrt{\omega} \mathbf{v}(\mathbf{k})^{*} \cdot \tilde{\partial}_{j}(\sqrt{\omega} \mathbf{v}(\mathbf{k}))+c t P_{j}, \\
\tilde{\partial}_{j} & \equiv \frac{\partial}{\partial k_{j}} .
\end{aligned}
$$

At this point, $\mathbf{v}(\mathbf{k})$ is usually expanded in terms of a basis of two polarization vectors $\boldsymbol{\epsilon}_{\alpha}(\mathbf{k}), \alpha=1,2$, obeying

$$
\begin{gathered}
\mathbf{k} \cdot \boldsymbol{\epsilon}_{\alpha}(\mathbf{k})=0, \quad \boldsymbol{\epsilon}_{\alpha}(\mathbf{k})^{*} \cdot \boldsymbol{\epsilon}_{\beta}(\mathbf{k})=\delta_{\alpha \beta}, \\
\boldsymbol{\epsilon}_{1}(\mathbf{k}) \wedge \boldsymbol{\epsilon}_{2}(\mathbf{k})=\hat{\mathbf{k}}
\end{gathered}
$$

as

$$
\mathbf{v}(\mathbf{k})=\sum_{\alpha=1}^{2} \boldsymbol{\epsilon}_{\alpha}(\mathbf{k}) v_{\alpha}(\mathbf{k})
$$

In general we can allow $\boldsymbol{\epsilon}_{\alpha}(\mathbf{k})$ to depend on $\mathbf{k}$, though sometimes we may assume dependence on $\hat{\mathbf{k}}$ alone. This will be clear from the context.

However, there are subtleties associated with choosing such polarization bases in a globally smooth way as $\hat{\mathbf{k}}$ varies over all possible directions, so we take this up later $[26$ - 28

We now move from real $\mathbf{A}, \mathbf{E}$ to their complex analytic signals $\mathbf{A}^{(+)}, \mathbf{E}^{(+)}$. For the definition and properties of analytic signals see, for instance [2, 29]. This gives the classical theory a form similar to quantum expressions. The analytic signals are the positive frequency parts of the real fields:

$$
\begin{aligned}
& \mathbf{E}=\mathbf{E}^{(+)}+\mathbf{E}^{(-)}=2 \operatorname{Re} \mathbf{E}^{(+)}, \\
& \mathbf{A}=\mathbf{A}^{(+)}+\mathbf{A}^{(-)}=2 \operatorname{Re} \mathbf{A}^{(+)} .
\end{aligned}
$$

While eqs. (2.8) are obeyed after the replacements $\mathbf{A}, \mathbf{E}, \mathbf{B} \rightarrow \mathbf{A}^{(+)}, \mathbf{E}^{(+)}, \mathbf{B}^{(+)}$, we also have first order EOM in time which are nonlocal in space:

$$
\begin{aligned}
i \frac{\partial}{\partial t} \mathbf{A}^{(+)}(\mathbf{x}, t) & =\left(\hat{\omega} \mathbf{A}^{(+)}\right)(\mathbf{x}, t), \\
i \frac{\partial}{\partial t} \mathbf{E}^{(+)}(\mathbf{x}, t) & =\left(\hat{\omega} \mathbf{E}^{(+)}\right)(\mathbf{x}, t), \\
\hat{\omega} & =c\left(-\nabla^{2}\right)^{1 / 2}
\end{aligned}
$$

The general solution (2.132.14) becomes

$$
\begin{aligned}
& \mathbf{A}^{(+)}(\mathbf{x}, t)=\frac{c}{2 \pi} \int \frac{d^{3} k}{\sqrt{\omega}} e^{i k \cdot x} \mathbf{v}(\mathbf{k}) \\
& \mathbf{E}^{(+)}(\mathbf{x}, t)=\frac{i}{2 \pi} \int d^{3} k \sqrt{\omega} e^{i k \cdot x} \mathbf{v}(\mathbf{k}) .
\end{aligned}
$$

The COM's (2.12) can all be written in terms of analytic signals. We need to use the hermiticity of $\hat{\omega}$ in the sense that for suitable complex $\psi(\mathbf{x}), \phi(\mathbf{x})$ we have

$$
\int d^{3} x \phi(\mathbf{x})^{*}(\hat{\omega} \psi)(\mathbf{x})=\int d^{3} x((\hat{\omega} \phi)(\mathbf{x}))^{*} \psi(\mathbf{x}) .
$$

Then after some algebra we find:

$$
\begin{aligned}
P^{0} & =\frac{1}{2 \pi} \int d^{3} x \mathbf{E}^{(+)}(x)^{*} \cdot \partial^{0} \mathbf{A}^{(+)}(x), \\
P_{j} & =\frac{1}{2 \pi c} \int d^{3} x \mathbf{E}^{(+)}(x)^{*} \cdot \partial_{j} \mathbf{A}^{(+)}(x), \\
J_{j}= & \frac{1}{2 \pi c} \int d^{3} x E_{m}^{(+)}(x)^{*}\left(\delta_{m n}(\mathbf{x} \wedge \nabla)_{j}\right. \\
\left.\quad+\epsilon_{j m n}\right) A_{n}^{(+)}(x), & \\
K_{j}(t)= & \frac{i}{2 \pi c^{2}} \int d^{3} x \mathbf{E}^{(+)}(x)^{*} \cdot\left(x_{j} \hat{\omega} \mathbf{A}^{(+)}\right)(x) .
\end{aligned}
$$

Thus eqs. (2.12/2.222.15) give $P^{\mu}, \mathbf{J}, \mathbf{K}(t)$ in terms of real fields, complex analytic signals, and $\mathbf{k}$-space amplitudes respectively.

In the real formulation, $\mathbf{E}(\mathbf{x}, 0)$ and $\mathbf{A}(\mathbf{x}, 0)$ can be chosen independently, then eqs. (2.8) determine $\mathbf{E}(x), \mathbf{A}(x)$ for all $t$. In the complex formulation, only $\mathbf{A}^{(+)}(\mathbf{x}, 0)$ can be chosen independently. Then both $\mathbf{A}^{(+)}(x)$ and $\mathbf{E}^{(+)}(x)=\partial^{0} \mathbf{A}^{(+)}(x)=\frac{i}{c}\left(\hat{\omega} \mathbf{A}^{(+)}\right)(x)$ are determined by eq. (2.19).

The structure of the total angular momentum $\mathbf{J}$ in eq. (2.22) has led to the suggestion that we regard it as the sum of 'orbital' and 'spin' parts $\mathbf{L}$ and $\mathbf{S}$, each real, defined as [30]

$$
\begin{aligned}
L_{j} & =\frac{1}{2 \pi c} \int d^{3} x E_{m}^{(+)}(x)^{*}(\mathbf{x} \wedge \nabla)_{j} A_{m}^{(+)}(x), \\
S_{j} & =\frac{1}{2 \pi c} \int d^{3} x \epsilon_{j m n} E_{m}^{(+)}(x)^{*} A_{n}^{(+)}(x) .
\end{aligned}
$$

From the first order EOM (2.19), the hermiticity (2.21) of $\hat{\omega}$, and the commutativity of $\hat{\omega}$ and $\mathbf{x} \wedge \nabla$, we see that both $\mathbf{L}$ and $\mathbf{S}$ are COM's. Since they are unambiguously defined expressions, they are legitimate classical dynamical variables.

The forms of the expressions (2.22) suggest that we introduce a complex Hilbert space at the classical level as follows. Guided by eq. (2.21) we initially define a natural looking inner product among complex (transverse) vector fields $\mathbf{V}(\mathbf{x}), \mathbf{V}^{\prime}(\mathbf{x}), \ldots$ on $\mathbb{R}^{3}$ as

$$
\left(\mathbf{V}^{\prime}(\cdot), \mathbf{V}(\cdot)\right)_{0}=\int d^{3} x \mathbf{V}^{\prime}(\mathbf{x})^{*} \cdot \mathbf{V}(\mathbf{x})
$$

Then we find

$$
\begin{aligned}
\left(\mathbf{V}^{\prime}(\cdot), \hat{\omega} \mathbf{V}(\cdot)\right)_{0}= & \left(\hat{\omega} \mathbf{V}^{\prime}(\cdot), \mathbf{V}(\cdot)\right)_{0} ; \\
\left(\mathbf{V}^{\prime}(\cdot), \boldsymbol{\alpha} \cdot \mathbf{x} \wedge \boldsymbol{\nabla} \mathbf{V}(\cdot)\right)_{0}= & -\left(\boldsymbol{\alpha} \cdot \mathbf{x} \wedge \nabla \mathbf{V}^{\prime}(\cdot), \mathbf{V}(\cdot)\right)_{0}, \\
& \boldsymbol{\alpha} \in \mathbb{R}^{3} .
\end{aligned}
$$

From given analytic signal solutions $\mathbf{A}^{(+)}(x), \mathbf{E}^{(+)}(x)$ to the Maxwell equations we can form three inner products $\left(\mathbf{A}^{(+)}(\cdot, t), \mathbf{A}^{(+)}(\cdot, t)\right)_{0},\left(\mathbf{E}^{(+)}(\cdot, t), \mathbf{A}^{(+)}(\cdot, t)\right)_{0}$ and 
$\left(\mathbf{E}^{(+)}(\cdot, t), \mathbf{E}^{(+)}(\cdot, t)\right)_{0}$. From eq. (2.25a) all of them are time independent. However we now show that only the second one is also Lorentz invariant.

The separations (2.18) of $\mathbf{A}(x), \mathbf{E}(x)$ into positive and negative frequency parts are Lorentz invariant. Therefore from eqs. (2.9) we can read off the changes in functional forms of $\mathbf{A}^{(+)}(x), \mathbf{E}^{(+)}(x)$ under an infinitesimal Lorentz transformation:

$$
\begin{aligned}
\delta \mathbf{A}^{(+)}(x) & \equiv \mathbf{A}^{(+) \prime}(x)-\mathbf{A}^{(+)}(x) \\
& =t \mathbf{v} \cdot \boldsymbol{\nabla} \mathbf{A}^{(+)}(x)-\frac{\mathbf{v} \cdot \mathbf{x}}{c} \mathbf{E}^{(+)}(x) \\
& +\frac{1}{c} \boldsymbol{\nabla}\left(\frac{1}{\nabla^{2}} \mathbf{v} \cdot \mathbf{E}^{(+)}\right)(x), \\
\delta \mathbf{E}^{(+)}(x) & \equiv \mathbf{E}^{(+) \prime}(x)-\mathbf{E}^{(+)}(x) \\
& =t \mathbf{v} \cdot \boldsymbol{\nabla} \mathbf{E}^{(+)}(x)-\frac{\mathbf{v} \cdot \mathbf{x}}{c} \nabla^{2} \mathbf{A}^{(+)}(x) \\
& +\frac{1}{c} \mathbf{v} \wedge \mathbf{B}^{(+)}(x) .
\end{aligned}
$$

Then, using transversality and dropping surface terms, we find:

$$
\begin{aligned}
\delta & \left(\mathbf{E}^{(+)}(\cdot, t), \mathbf{A}^{(+)}(\cdot, t)\right)_{0} \\
= & \left(\delta \mathbf{E}^{(+)}(\cdot, t), \mathbf{A}^{(+)}(\cdot, t)\right)_{0}+\left(\mathbf{E}^{(+)}(\cdot, t), \delta \mathbf{A}^{(+)}(\cdot, t)\right)_{0} \\
= & \frac{1}{c} \int d^{3} x \mathbf{v} \wedge \mathbf{B}^{(+)}(x)^{*} \cdot \mathbf{A}^{(+)}(x) \\
& +\frac{i}{c^{2}} \int d^{3} x \mathbf{E}^{(+)}(x)^{*} \cdot\left([\hat{\omega}, \mathbf{v} \cdot \mathbf{x}] \mathbf{A}^{(+)}\right)(x) \\
= & \frac{1}{c} \int d^{3} x \mathbf{v} \wedge\left(\boldsymbol{\nabla} \wedge \mathbf{A}^{(+)}(x)\right)^{*} \cdot \mathbf{A}^{(+)}(x) \\
& -i \int d^{3} x \mathbf{E}^{(+)}(x)^{*} \cdot\left(\mathbf{v} \cdot \nabla \frac{1}{\hat{\omega}} \mathbf{A}^{(+)}\right)(x) \\
= & \frac{1}{c} \int d^{3} x\left(\nabla \mathbf{v} \cdot \mathbf{A}^{(+)}(x)-\mathbf{v} \cdot \boldsymbol{\nabla} \mathbf{A}^{(+)}(x)\right)^{*} \cdot \mathbf{A}^{(+)}(x) \\
& -\frac{1}{c} \int d^{3} x\left(\hat{\omega} \mathbf{A}^{(+)}\right)(x)^{*} \cdot\left(\mathbf{v} \cdot \nabla \frac{1}{\hat{\omega}} \mathbf{A}^{(+)}\right)(x) \\
= & 0 .
\end{aligned}
$$

Here we used the operator relations

$$
\left[\hat{\omega}^{2}, \mathbf{x}\right]=-2 c^{2} \boldsymbol{\nabla}, \quad[\hat{\omega}, \mathbf{x}]=-c^{2} \frac{\boldsymbol{\nabla}}{\hat{\omega}} .
$$

Therefore $\left(\mathbf{E}^{(+)}(\cdot, t), \mathbf{A}^{(+)}(\cdot, t)\right)_{0}$ is both time independent and Lorentz invariant.

We point out that for real $\mathbf{E}, \mathbf{A}$ the expression $\int d^{3} x \mathbf{E}(x) \cdot \mathbf{A}(x)$ is neither Lorentz invariant nor time independent. Both these facts can be traced to the freedom to choose $\mathbf{E}(\mathbf{x}, 0)$ and $\mathbf{A}(\mathbf{x}, 0)$ independently as initial data. This emphasizes the advantages of using analytic signals.

Based on these considerations we define a Lorentz invariant and time independent squared norm for any an- alytic signal as

$$
\begin{aligned}
& \left(\mathbf{A}^{(+)}(\cdot, t), \mathbf{A}^{(+)}(\cdot, t)\right) \\
& =\frac{i}{2 \pi c}\left(\mathbf{E}^{(+)}(\cdot, t), \mathbf{A}^{(+)}(\cdot, t)\right)_{0} \\
& =\frac{1}{2 \pi c^{2}}\left(\mathbf{A}^{(+)}(\cdot, t), \hat{\omega} \mathbf{A}^{(+)}(\cdot, t)\right)_{0} \\
& =\frac{1}{2 \pi c^{2}} \int d^{3} x \mathbf{A}^{(+)}(x)^{*} \cdot\left(\hat{\omega} \mathbf{A}^{(+)}\right)(x) \geq 0 .
\end{aligned}
$$

Since

$$
\left(\mathbf{A}^{(+)}(\cdot, t), \hat{\omega} \mathbf{A}^{(+)}(\cdot, t)\right)_{0}=2 \pi c^{2} \int d^{3} k \mathbf{v}(\mathbf{k})^{*} \cdot \mathbf{v}(\mathbf{k}),
$$

we are led to a complex Hilbert space $\mathcal{M}$ at the classical level. This Hilbert space at the classical level has been used in [31] It has also been used in [23] where however, its Lorentz invariance has not been discussed or proved.

$$
\begin{aligned}
\mathcal{M} & =\left\{\mathbf{A}^{(+)}(x)\left\|\mathbf{A}^{(+)}\right\|^{2}=\left(\mathbf{A}^{(+)}(\cdot, t), \mathbf{A}^{(+)}(\cdot, t)\right)<\infty\right\} \\
& =\left\{\mathbf{v}(\mathbf{k}) \mid \mathbf{k} \cdot \mathbf{v}(\mathbf{k})=0,\|\mathbf{v}\|^{2}\right. \\
& \left.=\int d^{3} k \mathbf{v}(\mathbf{k})^{*} \cdot \mathbf{v}(\mathbf{k})<\infty\right\} .
\end{aligned}
$$

We can think of either $\mathbf{A}^{(+)}(x)$ or $\mathbf{v}(\mathbf{k})$ connected by eq. (2.20) as specifying an element of $\mathcal{M}$. The usefulness of this construction will emerge as we proceed to the quantized Maxwell field and the quantum mechanics of single photons. Both of eqs. (2.25) remain valid with the new inner product:

$$
\begin{aligned}
& \left(\mathbf{A}^{(+) \prime}(\cdot, t), \hat{\omega} \mathbf{A}^{(+)}(\cdot, t)\right) \\
& =\left(\hat{\omega} \mathbf{A}^{(+) \prime}(\cdot, t), \mathbf{A}^{(+)}(\cdot, t)\right) \\
& \left(\mathbf{A}^{(+) \prime}(\cdot, t), \boldsymbol{\alpha} \cdot \mathbf{x} \wedge \nabla \mathbf{A}^{(+)}(\cdot, t)\right) \\
& =-\left(\boldsymbol{\alpha} \cdot \mathbf{x} \wedge \nabla \mathbf{A}^{(+) \prime}(\cdot, t), \mathbf{A}^{(+)}(\cdot, t)\right) .
\end{aligned}
$$

\section{THE QUANTIZED RADIATION FIELD, OPERATOR COM'S, QUANTUM MECHANICS OF SINGLE PHOTONS}

In this Section we briefly recall the canonical quantization of the Maxwell field, and study the properties of the basic operator COM's and the counterparts of $\mathbf{L}$ and $\mathbf{S}$ of eq. (2.23). We then look at some features of single photon states and operator actions on them. 


\section{Quantization and basic operator COM's}

Since the free Maxwell equations (2.8) are linear, we can first construct their general solution and then perform quantization. In the analytic signal formulation, the general solution (2.20) involves the complex transverse vector amplitude $\mathbf{v}(\mathbf{k})$. Consistent with transversality the Hamiltonian form of the classical theory contains the basic Poisson Bracket (PB) relations

$$
\begin{aligned}
& \left\{v_{j}(\mathbf{k}), v_{l}\left(\mathbf{k}^{\prime}\right)^{*}\right\}=-i\left(\delta_{j l}-\frac{k_{j} k_{l}}{|\mathbf{k}|^{2}}\right) \delta^{(3)}\left(\mathbf{k}-\mathbf{k}^{\prime}\right) \\
& \{\mathbf{v}, \mathbf{v}\}=\left\{\mathbf{v}^{*}, \mathbf{v}^{*}\right\}=0
\end{aligned}
$$

Using the Dirac prescription 'quantum commutators' $\sim i \hbar$ 'classical PB's', we arrive at the canonical commutation relations (CCR) in a convenient form:

$$
\begin{aligned}
& \mathbf{v}(\mathbf{k}) \rightarrow \sqrt{\hbar} \hat{\mathbf{a}}(\mathbf{k}), \quad \mathbf{v}(\mathbf{k})^{*} \rightarrow \sqrt{\hbar} \hat{\mathbf{a}}(\mathbf{k})^{\dagger}: \\
& {\left[\hat{a}_{j}(\mathbf{k}), \hat{a}_{l}\left(\mathbf{k}^{\prime}\right)^{\dagger}\right]=\left(\delta_{j l}-\frac{k_{j} k_{l}}{|\mathbf{k}|^{2}}\right) \delta^{(3)}\left(\mathbf{k}-\mathbf{k}^{\prime}\right),} \\
& {[\hat{\mathbf{a}}, \hat{\mathbf{a}}]=\left[\hat{\mathbf{a}}^{\dagger}, \hat{\mathbf{a}}^{\dagger}\right]=0,} \\
& \mathbf{k} \cdot \hat{a}(\mathbf{k})=\mathbf{k} \cdot \hat{a}(\mathbf{k})^{\dagger}=0 .
\end{aligned}
$$

As with eqs. (2.16, 2.17) the transversality conditions can be accommodated by expanding $\hat{\mathbf{a}}(\mathbf{k}), \hat{\mathbf{a}}(\mathbf{k})^{\dagger}$ in terms of $\epsilon_{\alpha}(\mathbf{k})$ :

$$
\begin{aligned}
& \hat{\mathbf{a}}(\mathbf{k})=\sum_{\alpha=1}^{2} \boldsymbol{\epsilon}_{\alpha}(\mathbf{k}) \hat{a}_{\alpha}(\mathbf{k}), \\
& \hat{\mathbf{a}}(\mathbf{k})^{\dagger}=\sum_{\alpha=1}^{2} \boldsymbol{\epsilon}_{\alpha}(\mathbf{k})^{*} \hat{a}_{\alpha}(\mathbf{k})^{\dagger} ; \\
& {\left[\hat{a}_{\alpha}(\mathbf{k}), \hat{a}_{\beta}\left(\mathbf{k}^{\prime}\right)^{\dagger}\right]=\delta_{\alpha \beta} \delta^{(3)}\left(\mathbf{k}-\mathbf{k}^{\prime}\right),} \\
& {[\hat{a}, \hat{a}]=\left[\hat{a}^{\dagger}, \hat{a}^{\dagger}\right]=0 .}
\end{aligned}
$$

We mention again that there are subtleties involved in making globally smooth choices of such polarization bases which have been properly analyzed only recently 26 28]. It has of course been known that if one limits oneself to, say, linear polarizations, no globally smooth choices exist. Once one allows general polarization states with complex $\boldsymbol{\epsilon}_{\alpha}(\mathbf{k})$ 's, global smoothness can be achieved. We introduce chosen polarization bases later when needed.

The space-time dependent operator analytic signals and hermitian field operators are:

$$
\begin{aligned}
& \hat{\mathbf{A}}^{(+)}(x)=\frac{c}{2 \pi} \sqrt{\hbar} \int \frac{d^{3} k}{\sqrt{\omega}} e^{i k \cdot x} \hat{\mathbf{a}}(\mathbf{k}), \\
& \hat{\mathbf{E}}^{(+)}(x)=\frac{i}{2 \pi} \sqrt{\hbar} \int d^{3} k \sqrt{\omega} e^{i k \cdot x} \hat{\mathbf{a}}(\mathbf{k}) ; \\
& \hat{\mathbf{A}}(x)=\hat{\mathbf{A}}^{(+)}(x)+\hat{\mathbf{A}}^{(-)}(x), \\
& \hat{\mathbf{E}}(x)=\hat{\mathbf{E}}^{(+)}(x)+\hat{\mathbf{E}}^{(-)}(x) .
\end{aligned}
$$

The Hilbert space $\mathcal{H}$ on which all the above operators act and realize their CR's is the direct sum of subspaces labelled by the total photon number $n=0,1,2, \ldots$ :

$$
\mathcal{H}=\sum_{\oplus n=0}^{\infty} \mathcal{H}_{n}
$$

$\mathcal{H}_{0}$ is spanned by the vacuum state $|0\rangle$ with no photons:

$$
\begin{aligned}
& \mathcal{H}_{0}=\{\lambda|0\rangle, \quad \lambda \in \mathbb{C}\}, \\
& \hat{\mathbf{a}}(\mathbf{k})|0\rangle=0, \quad\langle 0 \mid 0\rangle=1 .
\end{aligned}
$$

The subspace $\mathcal{H}_{1}$ consists of all single photon states, obtained by applying all possible (normalisable) 'linear combinations' of $\hat{\mathbf{a}}(\mathbf{k})^{\dagger}$ to $|0\rangle$ :

$$
\mathcal{H}_{1}=\operatorname{Sp}\left\{\hat{a}_{j}(\mathbf{k})^{\dagger}|0\rangle \mid \mathbf{k} \in \mathbb{R}^{3}, j=1,2,3\right\} .
$$

Action by $\hat{\mathbf{a}}(\mathbf{k})^{\dagger}$ 's on $\mathcal{H}_{1}$ leads to the two-photon subspace $\mathcal{H}_{2}$, and so on.

The basic classical COM's written in eq. (2.22) in terms of analytic signals give immediately the hermitian quantum operator COM's in normal ordered form. They generate the unitary operators representing elements of the Poincaré group in the quantum theory. We list all of them including the pure Lorentz transformation generators:

$$
\begin{aligned}
\hat{P}_{0} & =\frac{i}{2 \pi c} \int d^{3} x \hat{\mathbf{E}}^{(+)}(x)^{\dagger} \cdot\left(\hat{\omega} \hat{\mathbf{A}}^{(+)}\right)(x) \\
& =\int d^{3} k \hbar \omega \hat{\mathbf{a}}(\mathbf{k})^{\dagger} \cdot \hat{\mathbf{a}}(\mathbf{k}) \\
\hat{P}_{j} & =\frac{1}{2 \pi c} \int d^{3} x \hat{\mathbf{E}}^{(+)}(x)^{\dagger} \cdot \partial_{j} \hat{\mathbf{A}}^{(+)}(x) \\
& =\int d^{3} k \hbar k_{j} \hat{\mathbf{a}}(\mathbf{k})^{\dagger} \cdot \hat{\mathbf{a}}(\mathbf{k}) ; \\
\hat{J}_{j} & =\frac{1}{2 \pi c} \int d^{3} x \hat{E}_{m}^{(+)}(x)^{\dagger}\left(\delta_{m n}(\mathbf{x} \wedge \nabla)_{j}+\epsilon_{j m n}\right) \hat{A}_{n}^{(+)}(x) \\
& =-i \hbar \int d^{3} k \hat{a}_{m}(\mathbf{k})^{\dagger}\left(\delta_{m n}\left(\mathbf{k} \wedge \tilde{\nabla}_{j}+\epsilon_{j m n}\right) \hat{a}_{n}(\mathbf{k}) ;\right. \\
\hat{K}_{j}(t) & =\frac{i}{2 \pi c^{2}} \int d^{3} x \hat{\mathbf{E}}^{(+)}(x)^{\dagger} \cdot\left(x_{j} \hat{\omega} \hat{\mathbf{A}}^{(+)}\right)(x) \\
& =c t \hat{\mathrm{P}}_{j}+\frac{i \hbar}{c} \int d^{3} k \sqrt{\omega} \hat{a}_{m}(\mathbf{k})^{\dagger} \tilde{\partial}_{j}\left(\sqrt{\omega} \hat{a}_{m}(\mathbf{k})\right) .
\end{aligned}
$$

The two hermitian COM's $\hat{\mathbf{J}}$ and $\hat{\mathbf{K}}(0)$, as is well known, realize the Lie algebra of the homogeneous Lorentz group $\mathrm{SO}(3,1)$ :

$$
\begin{aligned}
& {\left[\hat{J}_{j}, \hat{J}_{l}\right]=i \hbar \epsilon_{j l m} \hat{J}_{m}, \quad\left[\hat{J}_{j}, \hat{K}_{l}(0)\right]=i \hbar \epsilon_{j l m} \hat{K}_{m}(0),} \\
& {\left[\hat{K}_{j}(0), \hat{K}_{l}(0)\right]=-i \hbar \epsilon_{j l m} \hat{J}_{m} .}
\end{aligned}
$$

Next we consider the quantum operator analogues of the proposed definitions, eqs. (2.23), of 'orbital' and 'spin' angular momenta $\mathbf{L}, \mathbf{S}$ for the free Maxwell field. From eqs. (3.8) let us define the operators

$$
\begin{aligned}
& \hat{L}_{j}=-i \hbar \int d^{3} k \hat{a}_{m}(\mathbf{k})^{\dagger}(\mathbf{k} \wedge \tilde{\nabla})_{j} \hat{a}_{m}(\mathbf{k}), \\
& \hat{S}_{j}=-i \hbar \int d^{3} k \hat{a}_{m}(\mathbf{k})^{\dagger} \epsilon_{j m n} \hat{a}_{n}(\mathbf{k}),
\end{aligned}
$$


SO

$$
\hat{J}_{j}=\hat{L}_{j}+\hat{S}_{j}
$$

Each of $\hat{L}_{j}$ and $\hat{S}_{j}$ is well-defined and hermitian (as well as a COM). Therefore each of them is a physically observable dynamical variable representing a corresponding property of the quantum field. However, whether they may be regarded as angular momenta in the quantum mechanical sense depends on their CR's. The analyses of $\hat{L}_{j}$ and $\hat{S}_{j}$ follow similar lines. We find after some algebra:

$$
\left[\hat{L}_{j}, \hat{L}_{l}\right]=i \hbar \epsilon_{j l m}\left(\hat{L}_{m}-\hat{S}_{m}\right)
$$

While the right hand side is antihermitian, the presence of the second term shows that the $\hat{L}_{j}$ are not a quantum mechanical angular momentum triplet. This is so despite each $\hat{L}_{j}$ being hermitian and a COM. Similarly the CR's among the $\hat{S}_{j}$ can be computed and are

$$
\left[\hat{S}_{j}, \hat{S}_{l}\right]=0
$$

Therefore the $\hat{S}_{j}$ too do not form a quantum mechanical angular momentum. These facts concerning $\hat{L}_{j}$ and $\hat{S}_{j}$ have been noted earlier 30]. They mean that a priori we cannot draw any conclusions about the eigenvalues of each $\hat{L}_{j}$ and each $\hat{S}_{j}$, or of $\hat{\mathbf{L}} \cdot \hat{\mathbf{L}}$ and $\hat{\mathbf{S}} \cdot \hat{\mathbf{S}}$. In fact, unlike with a true angular momentum, all the components $\hat{S}_{j}$ can be simultaneously diagonalised.

To complete the picture, we need the commutators $\left[\hat{S}_{j}, \hat{L}_{l}\right]$ which turn out to be

$$
\left[\hat{S}_{j}, \hat{L}_{l}\right]=i \hbar \epsilon_{j l m} \hat{S}_{m}
$$

We infer: (i) since these commutators are nonzero, $\hat{\mathbf{L}}$ and $\hat{\mathbf{S}}$ are not kinematically independent; (ii) by combining eqs. (3.12, 3.13, 3.14) we obtain

$$
\left[\hat{J}_{j}, \hat{L}_{l} \quad \text { or } \quad \hat{S}_{l}\right]=i \hbar \epsilon_{j l m}\left(\hat{L}_{m} \text { or } \hat{S}_{m}\right) \text {. }
$$

Therefore both $\hat{\mathbf{L}}$ and $\hat{\mathbf{S}}$ transform as vectors under spatial $\mathrm{SO}(3)$ rotations generated by $\hat{\mathbf{J}}$.

It is worth noting that eqs. 3.15 mean that the following would have been equivalent: $\hat{\mathbf{L}}$ obeys the angular momentum CR's; $\hat{\mathbf{S}}$ obeys these CR's; $\hat{\mathbf{S}}$ and $\hat{\mathbf{L}}$ commute with one another. These are the properties characteristic of orbital and spin angular momenta for massive particles. Of course, none of these is true. On the other hand, we see that $\hat{\mathbf{J}}$ and $\hat{\mathbf{S}}$ obey the CR's corresponding to the Euclidean group E(3), though each of them has the dimensions of action.

\section{Single photon states - properties, operator actions}

The one-photon subspace $\mathcal{H}_{1}$ is defined in eq. (3.7). There is a natural and direct correspondence between the classical Hilbert space $\mathcal{M}$ of eq. (2.31) and $\mathcal{H}_{1}$. For each $\mathbf{v}(\cdot) \in \mathcal{M}$ with classical analytic signal $\mathbf{A}^{(+)}(x)$, we define a corresponding creation - annihilation operator pair $\hat{a}(\mathbf{v})^{\dagger}, \hat{a}(\mathbf{v})$ for photons 'in the state $\mathbf{v}(\cdot)$ '. Such operators have been introduced earlier in reference [31] as well as in reference [23].

$$
\begin{aligned}
& \mathbf{v}(\mathbf{k}) \in \mathcal{M}, \quad \mathbf{A}^{(+)}(\mathbf{x}, t)=\frac{c}{2 \pi} \int \frac{d^{3} k}{\sqrt{\omega}} e^{i k \cdot x} \mathbf{v}(\mathbf{k}) \rightarrow \\
& \hat{a}(\mathbf{v})=\frac{1}{\sqrt{\hbar}} \int d^{3} k \mathbf{v}(\mathbf{k})^{*} \cdot \hat{\mathbf{a}}(\mathbf{k}), \quad \hat{a}(\mathbf{v})^{\dagger} \\
& =\frac{1}{\sqrt{\hbar}} \int d^{3} k \mathbf{v}(\mathbf{k}) \cdot \hat{\mathbf{a}}(\mathbf{k})^{\dagger} ; \\
& {\left[\hat{a}(\mathbf{v}), \hat{a}\left(\mathbf{v}^{\prime}\right)^{\dagger}\right]=\frac{\left(\mathbf{v}, \mathbf{v}^{\prime}\right)}{\hbar} \mathbb{1} ;} \\
& |\mathbf{v}\rangle=\hat{a}(\mathbf{v})^{\dagger}|0\rangle \in \mathcal{H}_{1},\left\langle\mathbf{v}^{\prime} \mid \mathbf{v}\right\rangle=\frac{\left(\mathbf{v}^{\prime}, \mathbf{v}\right)}{\hbar}
\end{aligned}
$$

The connection to the classical analytic signal is through

$$
\left\langle 0\left|\hat{\mathbf{A}}^{(+)}(x)\right| \mathbf{v}\right\rangle=\mathbf{A}^{(+)}(x) .
$$

In this sense, every classical $\mathbf{v}(\mathbf{k})$ and associated $\mathbf{A}^{(+)}(x)$ are the momentum space and physical space wave functions respectively for a single photon in the state $|\mathbf{v}\rangle$. After normalization this state is

$$
\sqrt{\hbar} \frac{|\mathbf{v}\rangle}{\|\mathbf{v}\|}=\frac{1}{\|\mathbf{v}\|} \int d^{3} k \mathbf{v}(\mathbf{k}) \cdot \hat{\mathbf{a}}(\mathbf{k})^{\dagger}|0\rangle,
$$

so $\mathbf{v}(\mathbf{k})^{*} \cdot \mathbf{v}(\mathbf{k}) /\|\mathbf{v}\|^{2}$ is the $\mathbf{k}$-space probability density distribution. On the other hand, $\mathbf{A}^{(+)}(x)$ is the photon wave function in a formal sense, as $\mathbf{x}$ does not represent photon position.

Now we consider the actions of the operators $\hat{\mathbf{J}}, \hat{\mathbf{K}}(0), \hat{\mathbf{L}}$ and $\hat{\mathbf{S}}$, and of the infinitesimal unitary transformations generated by them, on single photon states. Each of these operators conserves total photon number, so it leaves invariant each subspace $\mathcal{H}_{n}$ in eq. (3.5). The restrictions of these operators to $\mathcal{H}_{1}$ (or to any $\mathcal{H}_{n}$ ) will therefore also obey the CR's (3.9, 3.12, 3.13, 3.14, 3.15). For simplicity we will use the same symbols for these restrictions as for the complete operators, as the meaning will be clear from the context. We take up $\hat{\mathbf{J}}, \hat{\mathbf{K}}(0)$ first.

From eqs. (3.8), using the CCR's (3.2), we obtain the CR's among $\hat{\mathbf{J}}_{j}, \hat{\mathbf{K}}_{j}(0)$ on the one hand, and $\hat{a}_{l}(\mathbf{k})^{\dagger}$ on the other. For $\hat{J}_{j}$ we get the set of results:

$$
\begin{array}{r}
{\left[\hat{J}_{j}, \hat{a}_{l}(\mathbf{k})^{\dagger}\right]=i \hbar\left(\delta_{l m}(\mathbf{k} \wedge \tilde{\nabla})_{j}+\epsilon_{j l m}\right) \hat{a}_{m}(\mathbf{k})^{\dagger}} \\
|\boldsymbol{\alpha}|<<1: \quad\left(1-\frac{i}{\hbar} \boldsymbol{\alpha} \cdot \hat{\mathbf{J}}\right)|\mathbf{v}\rangle \simeq|\mathbf{v}+\delta \mathbf{v}\rangle \\
\delta \mathbf{v}(\mathbf{k})=-\boldsymbol{\alpha} \cdot \mathbf{k} \wedge \tilde{\nabla} \mathbf{v}(\mathbf{k})+\boldsymbol{\alpha} \wedge \mathbf{v}(\mathbf{k})
\end{array}
$$

$$
\delta\left(\mathbf{v}(\mathbf{k})^{*} \cdot \mathbf{v}(\mathbf{k})\right)=\tilde{\nabla} \cdot\left(-\boldsymbol{\alpha} \wedge \mathbf{k} \mathbf{v}(\mathbf{k})^{*} \cdot \mathbf{v}(\mathbf{k})\right)
$$


Transversality is preserved, and the action is unitary. For $\hat{\mathbf{K}}_{j}(0)$ the analogous results are:

$$
\begin{aligned}
& {\left[\hat{K}_{j}(0), \hat{a}_{l}(\mathbf{k})^{\dagger}\right]=-i \frac{\hbar}{c} \sqrt{\omega}\left(\delta_{l m}-\frac{k_{l} k_{m}}{|\mathbf{k}|^{2}}\right) \tilde{\partial}_{j}\left(\sqrt{\omega} \hat{a}_{m}(\mathbf{k})^{\dagger}\right)} \\
& =-i \frac{\hbar}{c} \sqrt{\omega} \tilde{\partial}_{j}\left(\sqrt{\omega} \hat{a}_{l}(\mathbf{k})^{\dagger}\right)-\frac{i \hbar c}{\omega} k_{l} \hat{a}_{j}(\mathbf{k})^{\dagger} ; \\
& |\mathbf{v}|<<c: \quad\left(1-\frac{i}{\hbar c} \mathbf{v} \cdot \hat{\mathbf{K}}(0)\right)|\mathbf{v}\rangle \simeq|\mathbf{v}+\delta \mathbf{v}\rangle, \\
& \delta v_{l}(\mathbf{k})=\frac{1}{c^{2}}\left(\delta_{l m}-\frac{k_{l} k_{m}}{|\mathbf{k}|^{2}}\right) \sqrt{\omega} \mathbf{v} \cdot \tilde{\nabla}\left(\sqrt{\omega} v_{m}(\mathbf{k})\right) ; \\
& \delta\left(\mathbf{v}(\mathbf{k})^{*} \cdot \mathbf{v}(\mathbf{k})\right)=\tilde{\nabla} \cdot\left(\mathbf{v} \frac{\omega}{c^{2}} \mathbf{v}(\mathbf{k})^{*} \cdot \mathbf{v}(\mathbf{k})\right) .
\end{aligned}
$$

This expression for $\delta \mathbf{v}(\mathbf{k})$ agrees exactly with what one would get from eq. (2.26) for the change $\delta \mathbf{A}^{(+)}(\mathbf{x})$ in the vector potential caused by an infinitesimal Lorentz transformation, if re-expressed using eq. (2.20) as a change in $\mathbf{v}(\mathbf{k})$.

For later use we carry the discussion of $\hat{\mathbf{J}}$ a little further. Equation (3.19b) can be expressed as

$$
\boldsymbol{\alpha} \cdot \hat{\mathbf{J}} \mathbf{v}(\mathbf{k})=-i \hbar \boldsymbol{\alpha} \cdot \mathbf{k} \wedge \tilde{\nabla} \mathbf{v}(\mathbf{k})+i \hbar \boldsymbol{\alpha} \wedge \mathbf{v}(\mathbf{k})
$$

and in particular with $\mathbf{k}=k(\sin \theta \cos \varphi, \sin \theta \sin \varphi, \cos \theta)$,

$$
\hat{J}_{3} v_{j}(\mathbf{k})=-i \hbar \frac{\partial}{\partial \varphi} v_{j}(\mathbf{k})-i \hbar \epsilon_{3 j l} v_{l}(\mathbf{k})
$$

Since $\hat{\mathbf{J}}$ obeys the CR's in eq. (3.9), this infinitesimal action integrates to an action for finite rotations. The result is:

$$
\begin{aligned}
& \mathrm{e}^{-\frac{i}{\hbar} \boldsymbol{\alpha} \cdot \hat{\mathbf{J}}}|\mathbf{v}\rangle=\left|\mathbf{v}^{\prime}\right\rangle, \\
& \mathbf{v}^{\prime}(\mathbf{k})=R(\boldsymbol{\alpha})^{-1} \mathbf{v}(R(\boldsymbol{\alpha}) \mathbf{k}), \\
& R_{j l}(\boldsymbol{\alpha})=\delta_{j l} \cos \alpha+\alpha_{j} \alpha_{l} \frac{(1-\cos \alpha)}{\alpha^{2}}-\epsilon_{j l m} \alpha_{m} \frac{\sin \alpha}{\alpha} \\
& \alpha=|\boldsymbol{\alpha}| .
\end{aligned}
$$

The simultaneous eigenfunctions of $\hat{\mathbf{J}}^{2}$ and $\hat{J}_{3}$ (within $\left.\mathcal{H}_{1}\right)$ are well known [32, 33]. For each pair of integers $(l, m)$ with $l=1,2,3, \ldots,-l \leq m \leq l$, we have two orthonormal eigenfunctions depending on $\hat{\mathbf{k}}$ alone:

$$
\begin{aligned}
& \tilde{\mathbf{L}}=-i \mathbf{k} \wedge \tilde{\nabla}: \\
& \mathbf{Y}_{l m}^{(1)}(\hat{\mathbf{k}})=\frac{1}{\sqrt{l(l+1)}} \tilde{\mathbf{L}} Y_{l m}(\hat{\mathbf{k}}), \\
& \mathbf{Y}_{l m}^{(2)}(\hat{\mathbf{k}})=\frac{1}{\sqrt{l(l+1)}} \hat{\mathbf{k}} \wedge \tilde{\mathbf{L}} Y_{l m}(\hat{\mathbf{k}}) ; \\
& \left\{\hat{\mathbf{J}}^{2}, \hat{\mathbf{J}}_{3}\right\} \mathbf{Y}_{l m}^{(a)}(\hat{\mathbf{k}})=\left\{\hbar^{2} l(l+1), m \hbar\right\} \mathbf{Y}_{l m}^{(a)}(\hat{\mathbf{k}}), \\
& \quad a=1,2 ; \\
& \int_{\mathbb{S}^{2}} d \Omega(\hat{\mathbf{k}}) \mathbf{Y}_{l^{\prime} m^{\prime}}^{\left(a^{\prime}\right)}(\hat{\mathbf{k}})^{*} \cdot \mathbf{Y}_{l m}^{(a)}(\hat{\mathbf{k}})=\delta_{a^{\prime}, a} \delta_{l^{\prime}, l} \delta_{m^{\prime}, m} .
\end{aligned}
$$

(Here $Y_{l m}(\hat{\mathbf{k}})$ are the usual spherical harmonics). The two sets of eigenfunctions differ in their parity properties:

$\mathbf{Y}_{l m}^{(1)}(-\hat{\mathbf{k}})=(-1)^{l} \mathbf{Y}_{l m}^{(1)}(\hat{\mathbf{k}}), \quad \mathbf{Y}_{l m}^{(2)}(-\hat{\mathbf{k}})=(-1)^{l+1} \mathbf{Y}_{l m}^{(2)}(\hat{\mathbf{k}})$

A general $\mathbf{v}(\mathbf{k})$ has an expansion with two sets of 'radial' functions:

$$
\mathbf{v}(\mathbf{k})=\sum_{a=1}^{2} \sum_{l=1}^{\infty} \sum_{m=-l}^{l} f_{a, l m}(k) \mathbf{Y}_{l m}^{(a)}(\hat{\mathbf{k}}),
$$

and the squared norm (2.31) is

$(\mathbf{v}, \mathbf{v})=\int d^{3} k \mathbf{v}(\mathbf{k})^{*} \cdot \mathbf{v}(\mathbf{k})=\int_{0}^{\infty} k^{2} d k \sum_{a, l, m}\left|f_{a, l m}(k)\right|^{2}$

Finally we treat the operators $\hat{\mathbf{L}}, \hat{\mathbf{S}}$ in a similar manner. Following the pattern of eqs. (3.19), for $\hat{L}_{j}$ we have:

$$
\begin{aligned}
& {\left[\hat{L}_{j}, \hat{a}_{l}(\mathbf{k})^{\dagger}\right]=i \hbar\left\{(\mathbf{k} \wedge \tilde{\nabla})_{j} \hat{a}_{l}(\mathbf{k})^{\dagger}+\frac{k_{l}}{|\mathbf{k}|^{2}}\left(\mathbf{k} \wedge \hat{\mathbf{a}}(\mathbf{k})^{\dagger}\right)_{j}\right\} ;} \\
& |\boldsymbol{\alpha}|<<1: \quad\left(1-\frac{i}{\hbar} \boldsymbol{\alpha} \cdot \hat{\mathbf{L}}\right)|\mathbf{v}\rangle \simeq|\mathbf{v}+\delta \mathbf{v}\rangle, \\
& \delta \mathbf{v}(\mathbf{k})=-\boldsymbol{\alpha} \cdot(\mathbf{k} \wedge \tilde{\nabla}) \mathbf{v}(\mathbf{k})-\frac{\mathbf{k}}{|\mathbf{k}|^{2}} \boldsymbol{\alpha} \cdot \mathbf{k} \wedge \mathbf{v}(\mathbf{k}) ;(3.28 \mathrm{~b}) \\
& \delta\left(\mathbf{v}(\mathbf{k})^{*} \cdot \mathbf{v}(\mathbf{k})\right)=\tilde{\nabla} \cdot\left(\mathbf{k} \wedge \boldsymbol{\alpha} \mathbf{v}(\mathbf{k})^{*} \cdot \mathbf{v}(\mathbf{k})\right) .
\end{aligned}
$$

These too are consistent with transversality and unitarity.

The case of $\hat{\mathbf{S}}$ is particularly interesting, where we find:

$$
\begin{aligned}
& {\left[\hat{S}_{j}, \hat{a}_{l}(\mathbf{k})^{\dagger}\right]=i \hbar\left\{\epsilon_{j l m} \hat{a}_{m}(\mathbf{k})^{\dagger}-\frac{k_{l}}{|\mathbf{k}|^{2}}\left(\mathbf{k} \wedge \hat{\mathbf{a}}(\mathbf{k})^{\dagger}\right)_{j}\right\}} \\
& |\boldsymbol{\alpha}|<<1: \quad\left(1-\frac{i}{\hbar} \boldsymbol{\alpha} \cdot \hat{\mathbf{S}}\right)|\mathbf{v}\rangle \simeq|\mathbf{v}+\delta \mathbf{v}\rangle \\
& \delta \mathbf{v}(\mathbf{k})=\boldsymbol{\alpha} \wedge \mathbf{v}(\mathbf{k})-\frac{\mathbf{k}}{|\mathbf{k}|^{2}} \mathbf{k} \cdot \boldsymbol{\alpha} \wedge \mathbf{v}(\mathbf{k})=\boldsymbol{\alpha} \cdot \hat{\mathbf{k}} \hat{\mathbf{k}} \wedge \mathbf{v}(\mathbf{k})
\end{aligned}
$$

$$
\delta\left(\mathbf{v}(\mathbf{k})^{*} \cdot \mathbf{v}(\mathbf{k})\right)=0 .
$$

(In passing we note that eqs. (3.28a, 3.29a together give eq. (3.19a), and eqs (3.28b, 3.29b) give eq. (3.19b).) In contrast to the actions of $\hat{\mathbf{J}}, \hat{\mathbf{K}}(0)$ and $\hat{\mathbf{L}}$, in eqs. 3.29) there are no derivatives with respect to $\mathbf{k}$, the expression for $\delta \mathbf{v}(\mathbf{k})$ being purely algebraic in $\mathbf{v}(\mathbf{k})$. This makes the action of $\hat{\mathbf{S}}$ on $\mathbf{v}(\mathbf{k})$ much simpler. Using eq. (3.23) for general $R(\boldsymbol{\alpha}) \in S O(3)$, we have:

$$
|\boldsymbol{\alpha}| \ll 1: \quad \mathbf{v}(\mathbf{k})+\delta \mathbf{v}(\mathbf{k}) \simeq R(\boldsymbol{\alpha} \cdot \hat{\mathbf{k}} \hat{\mathbf{k}}) \mathbf{v}(\mathbf{k}) .
$$

Therefore at each $\hat{\mathbf{k}} \in \mathbb{S}^{2}, \mathbf{v}(\mathbf{k})$ experiences an infinitesimal right handed rotation by a variable $\hat{\mathbf{k}}$-dependent angle, $\boldsymbol{\alpha} \cdot \hat{\mathbf{k}}$, about the axis $\hat{\mathbf{k}}$. For general $\boldsymbol{\alpha} \in \mathbb{R}^{3}$, we have:

$$
\boldsymbol{\alpha} \cdot \hat{\mathbf{S}} \mathbf{v}(\mathbf{k})=i \hbar \boldsymbol{\alpha} \cdot \hat{\mathbf{k}} \hat{\mathbf{k}} \wedge \mathbf{v}(\mathbf{k})
$$


Taking a unit vector $\hat{\boldsymbol{\alpha}}$ for $\boldsymbol{\alpha}$, we then get

$$
(\hat{\boldsymbol{\alpha}} \cdot \hat{\mathbf{S}})^{2} \mathbf{v}(\mathbf{k})=\hbar^{2}(\hat{\boldsymbol{\alpha}} \cdot \hat{\mathbf{k}})^{2} \mathbf{v}(\mathbf{k}) .
$$

We can exhibit a link between $\hat{\mathbf{S}}$ and $\hat{\mathbf{J}}$ as well. From eqs. (3.21) we find

$$
\hat{\mathbf{k}} \cdot \hat{\mathbf{J}} \mathbf{v}(\mathbf{k})=i \hbar \hat{\mathbf{k}} \wedge \mathbf{v}(\mathbf{k})
$$

implying (on $\mathcal{H}_{1}$ !)

$$
\hat{\mathbf{S}}=\hat{\mathbf{k}} \hat{\mathbf{k}} \cdot \hat{\mathbf{J}}, \quad \hat{\mathbf{S}} \cdot \hat{\mathbf{S}}=\hbar^{2} .
$$

Since $\hat{\mathbf{k}}$ and $\hat{\mathbf{k}} \cdot \hat{\mathbf{J}}$ commute, the commutativity of the components $\hat{S}_{j}$ is obvious.

It can be seen from these considerations that for each $\hat{\boldsymbol{\alpha}}, \hat{\boldsymbol{\alpha}} \cdot \hat{\mathbf{S}}$ has continuous eigenvalues in the interval $(-\hbar, \hbar)$, so it has no normalisable eigenvectors. To simplify the action of $\hat{\boldsymbol{\alpha}} \cdot \hat{\mathbf{S}}$ on $\mathbf{v}(\mathbf{k})$ at each $\hat{\mathbf{k}}$, we need to introduce as a particular case of eqs. (2.16) an orthonormal pair of transverse 'circular polarization' vectors $\boldsymbol{\epsilon}^{(a)}(\mathbf{k}), a= \pm$, obeying:

$$
\begin{aligned}
& \boldsymbol{\epsilon}^{(a)}(\mathbf{k})^{*} \cdot \boldsymbol{\epsilon}^{(b)}(\mathbf{k})=\delta_{a, b} \\
& \hat{\mathbf{k}} \cdot \boldsymbol{\epsilon}^{(a)}(\mathbf{k})=0, \quad \boldsymbol{\epsilon}^{(-)}(\mathbf{k})=i \boldsymbol{\epsilon}^{(+)}(\mathbf{k})^{*} ; \\
& \hat{\mathbf{k}} \wedge \boldsymbol{\epsilon}^{( \pm)}(\mathbf{k})=\mp i \boldsymbol{\epsilon}^{( \pm)}(\mathbf{k}), \quad \boldsymbol{\epsilon}^{(+)}(\mathbf{k}) \wedge \boldsymbol{\epsilon}^{(-)}(\mathbf{k})=\hat{\mathbf{k}}
\end{aligned}
$$

If this were possible then we would have

$$
\hat{\boldsymbol{\alpha}} \cdot \hat{\mathbf{S}} \boldsymbol{\epsilon}^{( \pm)}(\mathbf{k})= \pm \hbar \hat{\boldsymbol{\alpha}} \cdot \hat{\mathbf{k}} \boldsymbol{\epsilon}^{( \pm)}(\mathbf{k}) .
$$

(These $\boldsymbol{\epsilon}^{(a)}(\mathbf{k})$ would not, however, be eigenvectors of $\hat{\boldsymbol{\alpha}} \cdot \hat{\mathbf{S}}$ in $\mathcal{H}_{1}$ !).

However such $\boldsymbol{\epsilon}^{( \pm)}(\mathbf{k})$ cannot be found in a globally smooth manner for all $\hat{\mathbf{k}} \in \mathbb{S}^{2}$. (If such a choice existed, then by eqs. (3.35) the real part of $\boldsymbol{\epsilon}^{(+)}(\mathbf{k})$ would be a nowhere vanishing globally smooth tangent vector to $\mathbb{S}^{2}$ at $\hat{\mathbf{k}}$ for all $\hat{\mathbf{k}} \in \mathbb{S}^{2}$ : but this is not allowed by the 'hairy ball' theorem whose proof is well known [34].) Fortunately, for applications to the paraxial situation in Section V, and for the physical interpretation of $\hat{\mathbf{S}}$ developed below, this does not matter. A choice well-defined everywhere except along the negative $z$-axis is:

$$
\begin{aligned}
& 0 \leq \theta<\pi, 0 \leq \varphi<2 \pi: \\
& \left\{\boldsymbol{\epsilon}^{(+)}(\mathbf{k}), \boldsymbol{\epsilon}^{(-)}(\mathbf{k})\right\} \\
& =R_{3}(\varphi) R_{2}(\theta) R_{3}(\varphi)^{-1}\left\{\frac{1}{\sqrt{2}}\left(\begin{array}{l}
1 \\
i \\
0
\end{array}\right), \frac{1}{\sqrt{2}}\left(\begin{array}{l}
i \\
1 \\
0
\end{array}\right)\right\} \\
& R_{3}(\varphi)=\left(\begin{array}{ccc}
\cos \varphi & -\sin \varphi & 0 \\
\sin \varphi & \cos \varphi & 0 \\
0 & 0 & 1
\end{array}\right) \\
& R_{2}(\theta)=\left(\begin{array}{ccc}
\cos \theta & 0 & \sin \theta \\
0 & 1 & 0 \\
-\sin \theta & 0 & \cos \theta
\end{array}\right)
\end{aligned}
$$

Expanding $\mathbf{v}(\mathbf{k})$ in this basis as

$$
\mathbf{v}(\mathbf{k})=\sum_{a= \pm} \boldsymbol{\epsilon}^{(a)}(\mathbf{k}) v_{a}(\mathbf{k})
$$

and using eqs. (3.30, 3.35) we see that

$$
\hat{S}_{j} v_{a}(\mathbf{k})=a \hbar \hat{k}_{j} v_{a}(\mathbf{k}), \quad a= \pm .
$$

Therefore in the normalized state $\sqrt{\hbar}|\mathbf{v}\rangle /\|\mathbf{v}\|$ the expectation value of $\hat{S}_{j}$ is

$$
\begin{aligned}
\frac{\hbar}{\|\mathbf{v}\|^{2}}\left\langle\mathbf{v}\left|\hat{S}_{j}\right| \mathbf{v}\right\rangle & =\hbar \int d^{3} k \hat{k}_{j}(p(\mathbf{k},+)-p(\mathbf{k},-)), \\
p(\mathbf{k}, a) & =\left|v_{a}(\mathbf{k})\right|^{2} /\|\mathbf{v}\|^{2},
\end{aligned}
$$

where $p(\mathbf{k}, \pm)$ are the probability densities in $\mathbf{k}$-space for the photon to have momentum $\hbar \mathbf{k}$ and to be right/left circularly polarized.

This discussion helps to bring out the meaning of the operators $\hat{S}_{j}$ as single photon observables. In particular the operators $\hat{S}_{j}$ (within single photon subspace) are completely defined by the expectation value expression (3.40) since it is given for all possible states. As is evident these operators commute with the momentum operator for the photon.

\section{THE PARAXIAL APPROXIMATION, RELATION TO QUANTIZATION PROCEDURE}

The treatment of the paraxial regime is an important part of classical ray as well as wave optics, which is important also for laser physics. In this Section we examine the problem of combining it in a physically reasonable way with the quantization of the radiation field outlined in the previous Section.

\section{Scalar paraxial case}

For simplicity we outline first the sequence of assumptions and approximations involved in paraxial optics (in leading order) in the scalar context, and then turn to the transverse vector potential. We are concerned with complex scalar analytic signal solutions $\psi(x)$ to the wave equation

$$
\left(\nabla^{2}-\frac{1}{c^{2}} \frac{\partial^{2}}{\partial t^{2}}\right) \psi(x)=0 .
$$

Expressing a general solution as a Fourier integral over positive frequencies,

$$
\psi(x)=\int_{0}^{\infty} d \omega \tilde{\psi}(\mathbf{x} ; \omega) e^{-i \omega t},
$$

the function $\tilde{\psi}$ obeys the free Helmholtz equation $(\omega=$ $c k=2 \pi c / \lambda)$

$$
\left(\nabla^{2}+k^{2}\right) \tilde{\psi}(\mathbf{x} ; \omega)=0
$$


The general solution of this equation, as a superposition of plane waves, is

$$
\tilde{\psi}(\mathbf{x} ; \omega)=\int_{\mathbb{S}^{2}} d \Omega(\hat{\mathbf{k}}) \phi(\mathbf{k}) e^{i \mathbf{k} \cdot \mathbf{x}}, \quad|\mathbf{k}|=k,
$$

where we may assume $\phi(\mathbf{k}) \in L^{2}\left(\mathbb{S}^{2}\right)$. Now we assume there are only 'forward propagating' waves in the sense that $\phi(\mathbf{k})$ vanishes for $k_{3}<0$. Then

$$
\begin{aligned}
& \tilde{\psi}(\mathbf{x} ; \omega)=\int_{\left|\mathbf{k}_{\perp}\right| \leq k} d^{2} \mathbf{k}_{\perp} \frac{\phi\left(\mathbf{k}_{\perp}, \sqrt{k^{2}-\mathbf{k}_{\perp}^{2}}\right)}{k \sqrt{k^{2}-\mathbf{k}_{\perp}^{2}}} \\
& \times e^{i \mathbf{k}_{\perp} \cdot \mathbf{x}_{\perp}+i \sqrt{k^{2}-\mathbf{k}_{\perp}{ }^{2}} z} .
\end{aligned}
$$

At this point we introduce the paraxial condition $\phi(\mathbf{k})$ is negligible unless $\left|\mathbf{k}_{\perp}\right| \ll k$ :

$$
\phi\left(\mathbf{k}_{\perp}, \sqrt{k^{2}-\mathbf{k}_{\perp}^{2}}\right) \approx 0 \text { unless }\left|\mathbf{k}_{\perp}\right|<<k .
$$

With this assumption we can carry out two simplifications: expand the square root in the exponent in eq. 4.5) to lowest nontrivial order, and formally extend the integration region to the entire plane:

$$
\begin{aligned}
\tilde{\psi}_{\operatorname{par}}(\mathbf{x} ; \omega) & \simeq e^{i k z} \psi_{0, \operatorname{par}}\left(\mathbf{x}_{\perp}, z ; \omega\right) \\
\psi_{0, \operatorname{par}}\left(\mathbf{x}_{\perp}, z ; \omega\right) & =\int_{\mathbb{R}^{2}} d^{2} \mathbf{k}_{\perp} \frac{\phi\left(\mathbf{k}_{\perp}, k\right)}{k^{2}} e^{i \mathbf{k}_{\perp} \cdot \mathbf{x}_{\perp}-i \frac{\hbar}{2} \mathbf{k}_{\perp}{ }^{2} z} .
\end{aligned}
$$

The amplitude $\psi_{0 \text {,par }}$ obeys the paraxial wave equation (PWE) for (reduced) wave length $\lambda=\lambda / 2 \pi$ :

$$
i \frac{\partial}{\partial z} \psi_{0, \operatorname{par}}\left(\mathbf{x}_{\perp}, z ; \omega\right)=-\frac{\lambda}{2} \nabla_{\perp}^{2} \psi_{0, \operatorname{par}}\left(\mathbf{x}_{\perp}, z ; \omega\right) .
$$

If we now try to retrace these steps to arrive at an approximate paraxial solution to the wave equation (4.1), we realize that for the condition (4.6) to make sense the range of frequencies involved in eq. (4.2) must be greater than some minimum $\omega_{\min }>0$. Incorporating this, we arrive at the following form for an approximate paraxial scalar analytic signal obeying eq. (4.1):

$$
\psi_{\mathrm{par}}(x) \simeq c \int_{k_{\min }}^{\infty} d k e^{i k(z-c t)} \psi_{0, \operatorname{par}}\left(\mathbf{x}_{\perp}, z ; \omega\right)
$$

with $\psi_{0, p a r}\left(\mathbf{x}_{\perp}, z ; \omega\right)$ given by eq. (4.7) and $\phi(\mathbf{k})$ obeying (4.6).

At this point it is important to realize that while $\psi_{0, p a r}\left(\mathbf{x}_{\perp}, z ; \omega\right)$ is a paraxial solution of the PWE (4.8), there are nonparaxial solutions as well. For the moment suppress $\omega$ as an argument. If at, say, $z=0$ we choose as 'initial' amplitude a general $\psi\left(\mathbf{x}_{\perp}, 0\right) \in L^{2}\left(\mathbb{R}_{\mathbf{x}_{\perp}}^{2}\right)$, and express it as

$$
\psi\left(\mathbf{x}_{\perp}, 0\right)=\int_{\mathbb{R}^{2}} d^{2} \mathbf{k}_{\perp} \phi\left(\mathbf{k}_{\perp}\right) e^{i \mathbf{k}_{\perp} \cdot \mathbf{x}_{\perp}},
$$

then $\phi\left(\mathbf{k}_{\perp}\right)$ is a general element of $L^{2}\left(\mathbb{R}_{\mathbf{k}_{\perp}}^{2}\right)$ and the solution of (4.8) is

$$
\psi\left(\mathbf{x}_{\perp}, z\right)=\int_{\mathbb{R}^{2}} d^{2} \mathbf{k}_{\perp} \phi\left(\mathbf{k}_{\perp}\right) e^{i \mathbf{k}_{\perp} \cdot \mathbf{x}_{\perp}-i \frac{\grave{\lambda}}{2} \mathbf{k}_{\perp}^{2} z} .
$$

This $\phi\left(\mathbf{k}_{\perp}\right)$ need not obey (4.6), so $\psi\left(\mathbf{x}_{\perp}, z\right)$ may not be paraxial at all. Thus most solutions to the PWE are nonparaxial.

To emphasize this fact, we take this discussion one step further. Choose any discrete orthonormal basis $\left\{\phi_{n}\left(\mathbf{k}_{\perp}\right)\right\}$ for $L^{2}\left(\mathbb{R}_{\mathbf{k}_{\perp}}^{2}\right)$ :

$$
\begin{gathered}
\int_{\mathbb{R}^{2}} d^{2} \mathbf{k}_{\perp} \phi_{n^{\prime}}\left(\mathbf{k}_{\perp}\right)^{*} \phi_{n}\left(\mathbf{k}_{\perp}\right)=\delta_{n^{\prime}, n}, \quad n^{\prime}, n=1,2, \cdots ; \\
\sum_{n=1}^{\infty} \phi_{n}\left(\mathbf{k}_{\perp}\right) \phi_{n}\left(\mathbf{k}_{\perp}^{\prime}\right)^{*}=\delta^{(2)}\left(\mathbf{k}_{\perp}-\mathbf{k}_{\perp}^{\prime}\right) .
\end{gathered}
$$

By Fourier transformation we get a corresponding orthonormal basis for $L^{2}\left(\mathbb{R}_{\mathbf{x}_{\perp}}^{2}\right)$, and a set of PWE solutions:

$$
\begin{gathered}
\psi_{n}\left(\mathbf{x}_{\perp}, 0\right)=\frac{1}{2 \pi} \int d^{2} \mathbf{k}_{\perp} \phi_{n}\left(\mathbf{k}_{\perp}\right) e^{i \mathbf{k}_{\perp} \cdot \mathbf{x}_{\perp}}, \\
\int_{\mathbb{R}^{2}} d^{2} \mathbf{x}_{\perp} \psi_{n^{\prime}}\left(\mathbf{x}_{\perp}, 0\right)^{*} \psi_{n}\left(\mathbf{x}_{\perp}, 0\right)=\delta_{n^{\prime}, n}, \\
\sum_{n=1}^{\infty} \psi_{n}\left(\mathbf{x}_{\perp}, 0\right) \psi_{n}\left(\mathbf{x}_{\perp}^{\prime}, 0\right)^{*}=\delta^{(2)}\left(\mathbf{x}_{\perp}-\mathbf{x}_{\perp}^{\prime}\right) ; \\
\psi_{n}\left(\mathbf{x}_{\perp}, z\right)=\frac{1}{2 \pi} \int_{\mathbb{R}^{2}} d^{2} \mathbf{k}_{\perp} \phi_{n}\left(\mathbf{k}_{\perp}\right) e^{i \mathbf{k}_{\perp} \cdot \mathbf{x}_{\perp}-i \frac{\pi}{2} \mathbf{k}_{\perp}{ }^{2} z} .
\end{gathered}
$$

For each $z,\left\{\psi_{n}\left(\mathbf{x}_{\perp}, z\right)\right\}$ is an orthonormal basis for $L^{2}\left(\mathbb{R}_{\mathbf{x}_{\perp}}^{2}\right)$. Combining eqs. (4.124.13a) also gives

$$
e^{i \mathbf{k}_{\perp} \cdot \mathbf{x}_{\perp}-i \frac{\hbar}{2} \mathbf{k}_{\perp}{ }^{2} z}=2 \pi \sum_{n=1}^{\infty} \phi_{n}\left(\mathbf{k}_{\perp}\right)^{*} \psi_{n}\left(\mathbf{x}_{\perp}, z\right) .
$$

Now a general initial $\psi\left(\mathbf{x}_{\perp}, 0\right)$, eq. 4.10), can be expanded as

$$
\psi\left(\mathbf{x}_{\perp}, 0\right)=\sum_{n=1}^{\infty} c_{n} \psi_{n}\left(\mathbf{x}_{\perp}, 0\right)
$$

where $\left\{c_{n}\right\}$ is an $l^{2}$-sequence,

$$
\sum_{n=1}^{\infty}\left|c_{n}\right|^{2}<\infty
$$

which is otherwise unrestricted. This initial $\psi\left(\mathbf{x}_{\perp}, 0\right)$ then evolves to the solution

$$
\psi\left(\mathbf{x}_{\perp}, z\right)=\sum_{n=1}^{\infty} c_{n} \psi_{n}\left(\mathbf{x}_{\perp}, z\right)
$$

to the PWE which may not be paraxial at all. If $\psi\left(\mathbf{x}_{\perp}, z\right)$ is to be a paraxial solution to the PWE, then $\sum_{n=1}^{\infty} c_{n} \phi_{n}\left(\mathbf{k}_{\perp}\right)$ must obey (4.6), which implies conditions on $\left\{c_{n}\right\}$ going well beyond the $l^{2}$ property (4.15). 


\section{Paraxial vector potential, route to quantization}

The general form of the complex analytic signal transverse vector potential is given in eq. (2.20) in terms of $\mathbf{v}(\mathbf{k})$. For $\mathbf{A}^{(+)}(x)$ to be paraxial, we see now that in addition to $\mathbf{k} \cdot \mathbf{v}(\mathbf{k})=0$, we must have

$$
\begin{array}{r}
\mathbf{v}\left(\mathbf{k}_{\perp}, k_{3}\right)=0 \text { for } k \leq k_{\min }, k_{3}<0 \\
\mathbf{v}\left(\mathbf{k}_{\perp}, \sqrt{k^{2}-\mathbf{k}_{\perp}^{2}}\right) \approx 0 \text { unless }\left|\mathbf{k}_{\perp}\right|<<k .
\end{array}
$$

Given such $\mathbf{v}(\mathbf{k})$, and following steps as in the scalar case, we get the following approximate form for a paraxial vector potential:

$$
\begin{aligned}
& \mathbf{A}_{\mathrm{par}}^{(+)}(x) \simeq \frac{c}{2 \pi} \int_{k_{\min }}^{\infty} k d k e^{i k(z-c t)} \int_{\mathbb{R}^{2}} \frac{d^{2} k_{\perp}}{\sqrt{\omega} \sqrt{k^{2}-\mathbf{k}_{\perp}^{2}}} \\
& \times \mathbf{v}\left(\mathbf{k}_{\perp}, k\right) e^{i \mathbf{k}_{\perp} \cdot \mathbf{x}_{\perp}-i \frac{\lambda}{2} \mathbf{k}_{\perp}{ }^{2} z} .
\end{aligned}
$$

Turning to 'quantization in the paraxial regime', to begin with it may seem reasonable to proceed as follows: start with the form (4.19) for the classical vector potential in this regime, then proceed to 'quantize' it by turning $\mathbf{A}_{\text {par }}^{(+)}(x)$ into a suitable operator $\hat{\mathbf{A}}_{\text {par }}^{(+)}(x)$. This would mean taking the paraxial approximation first, then performing quantization. For instance, in the spirit of eqs. (4.12 4.17), we may expand $\mathbf{A}_{\text {par }}^{(+)}(x)$ in some complete orthonormal set $\left\{\boldsymbol{\psi}_{n}\left(\mathbf{x}_{\perp}, 0\right)\right\}$, each member of which is paraxial, and obtain expansion coefficients similar to $\left\{c_{n}\right\}$ in eq. (4.15); and then convert them to operators as $c_{n} \rightarrow \hat{a_{n}}, c_{n}^{*} \rightarrow{\hat{a_{n}}}^{\dagger}$. However this procedure seems inadvisable for important physical reasons:

(i) The paraxial regime is defined only in an approximate way, as seen in eqs.4.6, 4.9, 4.18, 4.19). It is not possible to define it with any degree of mathematical precision, such as is associated with the quantization process.

(ii) In effect, only the classical amplitudes $\mathbf{v}\left(\mathbf{k}_{\perp}, k_{3}\right)$ for $k \geq k_{\text {min }}>0, k_{3}>0,\left|\mathbf{k}_{\perp}\right| \ll k$, would be converted into operators.

(iii) Even if this were possible, the classical condition (4.18) would have to be translated into statements on the resulting operators in some way, not however on the CCR's themselves. This is in essence the problem of restrictions on $\left\{c_{n}\right\}$ mentioned after eq. (4.17).

For these reasons, a better procedure seems to be as follows. We begin with the quantized radiation field as set up in Section III, in which with every classical analytic signal vector potential $\mathbf{A}^{(+)}(x)$ we are able to associate an (unnormalized) photon annihilation-creation operator pair $\hat{a}(\mathbf{v}), \hat{a}(\mathbf{v})^{\dagger}$ obeying eqs. (3.16). Here $\mathbf{v}(\mathbf{k})$ is any element of the classical Hilbert space $\mathcal{M}$, eq. (2.31). The operator $\hat{a}(\mathbf{v})^{\dagger}$ creates photons in the state, or with wave function, $\mathbf{v}(\mathbf{k})$. We then limit the choice of $\mathbf{v}(\mathbf{k})$ to those obeying the paraxial conditions (4.18), so we deal with a limited subset of the operator pairs $\hat{a}(\mathbf{v}), \hat{a}(\mathbf{v})^{\dagger}$. In this way, the paraxial conditions come after the quantization of the entire field, rather than the other way around. We accept the approximate nature of the statement of the paraxial conditions; and that $\mathbf{A}_{\text {par }}^{(+)}(x)$ of (4.19) obeys the wave equation only approximately. This understandable lack of precision gets expressed in the properties of the chosen $\mathbf{v}(\mathbf{k})$ 's, not in the quantization process. However, The procedure adopted here differs from that in [23], wherein an attempt is made to identify a subspace of $\mathcal{H}_{1}$, made up of paraxial wavefunctions. It would have to be checked if such a space is a closed as well as a proper subspace of $\mathcal{H}_{1}$. In reference [22], on the other hand, the paraxial limit is defined by an inequality $\theta<<1$ for a parameter $\theta$ that governs the degree of paraxiality, and quantization is done after this limit is taken in the classical vector potential. There is therefore a vagueness as to which original classical amplitudes are being made into operators.

In our approach, there is no paraxial vector potential field operator $\hat{\mathbf{A}}_{\text {par }}^{(+)}(x)$ at all. An examination of some properties of one photon states $|\mathbf{v}\rangle$ for paraxial $\mathbf{v}(\mathbf{k})$ is taken up in the next Section.

We conclude this Section by mentioning the LaguerreGaussian mode functions which are a particular widely used example of the complete orthonormal sets $\left\{\phi_{n}\left(\mathbf{k}_{\perp}\right)\right\},\left\{\psi_{n}\left(\mathbf{x}_{\perp}, z\right)\right\}$ in eqs. (4.12 4.14). They are characterized by a real waist parameter $w>0$; and the index $n=1,2, \ldots$ is replaced by a pair $(m, p), m=$ $0, \pm 1, \pm 2, \ldots$ and $p=0,1,2, \ldots$ independently. A readable account is available in reference [35].

(The index $m$ used here - the magnetic quantum number - is in the usual notation of the quantum theory of angular momentum (QTAM). It is often replaced by $l$ in the literature, which however has a different meaning in QTAM). Then the $\phi_{n}$ 's are, with $\mathbf{k}_{\perp}=\rho(\cos \varphi, \sin \varphi)$ :

$$
\begin{aligned}
\phi_{m, p}\left(\mathbf{k}_{\perp}\right)= & \frac{w}{\sqrt{2 \pi}} \sqrt{\frac{p !}{(p+|m|) !}} e^{i m \varphi}\left(\frac{i w \rho}{\sqrt{2}}\right)^{|m|} \\
& \times L_{p}^{|m|}\left(\frac{w^{2} \rho^{2}}{2}\right) e^{-\frac{w^{2} \rho^{2}}{4}}
\end{aligned}
$$

Here the $L$ 's are Laguerre polynomials. For the $\psi_{n}$ 's we need the expressions

$$
\begin{gathered}
w(\zeta)=w\left(1+\zeta^{2}\right)^{1 / 2}, \quad \zeta=z / z_{R} \\
z_{R}=w^{2} / 2 \lambda=\text { Rayleigh range. }
\end{gathered}
$$


Then with $\mathbf{x}_{\perp}=r(\cos \phi, \sin \phi)$, the $\psi_{n}$ 's are:

$$
\begin{aligned}
& \psi_{m, p}\left(\mathbf{x}_{\perp}, z\right)=\frac{(-1)^{p+|m|}}{w(\zeta)} \sqrt{\frac{2}{\pi} \frac{p !}{(p+|m|) !}} \\
& \times e^{-i(2 p+|m|+1) \tan ^{-1} \zeta} e^{i m \phi}\left(\frac{\sqrt{2} r}{w(\zeta)}\right)^{|m|} \\
& \times L_{p}^{|m|}\left(\frac{2 r^{2}}{w(\zeta)^{2}}\right) e^{-\frac{r^{2}}{w^{2}(1+i \zeta)}} .
\end{aligned}
$$

For any $w>0$, the $\psi_{m, p}\left(\mathbf{x}_{\perp}, z\right)$ are solutions of the PWE (4.8); to be paraxial solutions we must require $w \gg \lambda$. The identity (4.14) now reads:

$$
\begin{aligned}
e^{i \mathbf{k}_{\perp} \cdot \mathbf{x}_{\perp}-i \frac{\hbar}{2} \mathbf{k}_{\perp}^{2} z}= & 2 \pi \sum_{p=0,1, \cdots m=0, \pm 1, \cdots} \sum_{m, p}\left(\mathbf{k}_{\perp}\right)^{*} \\
& \times \psi_{m, p}\left(\mathbf{x}_{\perp}, z\right) .
\end{aligned}
$$

The Gaussian factor in $\phi_{m, p}\left(\mathbf{k}_{\perp}\right)$ ensures paraxiality but at different rates for different $m, p$. Any finite linear combination of the $\phi_{m, p}\left(\mathbf{k}_{\perp}\right)$ is also paraxial, so such a combination of $\psi_{m, p}\left(\mathbf{x}_{\perp}, z\right)$ is a paraxial solution of the PWE. However this is not necessarily true if we choose an otherwise unrestricted $l^{2}$-sequence $\left\{C_{m, p}\right\}$ and form the corresponding combination of $\psi_{m, p}\left(\mathbf{x}_{\perp}, z\right)$. This is an instance of the comments made after eq. (4.17).

We may repeat that the L-G mode functions are only one example of a complete orthonormal set along the lines of eqs. (4.12, 4.13), though important for practical purposes. While these functions have been recalled here in the scalar case, the extension to the transverse vector case is taken up in the next Section.

\section{VECTOR POTENTIAL AND SINGLE PHOTON STATES IN THE PARAXIAL REGIME}

As recalled in Section 3, the hermitian operators $\hat{\mathbf{L}}$ and $\hat{\mathbf{S}}$, unlike $\hat{\mathbf{J}}$, do not obey the CR's of a quantum mechanical angular momentum. Therefore, while from eq. (3.24b) the eigenvalues of $\hat{\mathbf{J}}^{2}, \hat{J}_{3}$ within $\mathcal{H}_{1}$ are known to have the familiar quantized forms $\hbar^{2} l(l+1), m \hbar$ with $l=1,2, \ldots$, and $-l \leq m \leq l$, there is no reason to expect similar patterns for the eigenvalues of $\hat{\mathbf{L}}^{2}, \hat{L}_{3}$ or $\hat{\mathbf{S}}^{2}, \hat{S}_{3}$. Indeed, we have found that the $\hat{S}_{j}$ can be simultaneously diagonalised and, within $\mathcal{H}_{1}$, each $\hat{S}_{j}$ has continuous eigenvalues in the interval $(-\hbar, \hbar)$ while obeying $\hat{S}_{j} \hat{S}_{j}=\hbar^{2}$.

Now, to carry forward the analysis of Section 4 and understand better the properties of the paraxial regime, we first examine the eigenfunctions of $\hat{J}_{3}$ within $\mathcal{H}_{1}$. We use spherical polar or cylindrical variables as convenient, and consider both $\mathbf{k}$-space and physical space expressions.
Forms of $\hat{J}_{3}$ eigenfunctions in $\mathcal{H}_{1}$

The action of $\hat{J}_{3}$ on a wave function $\mathbf{v}(\mathbf{k}) \in \mathcal{M}$ is given in eq. (3.22). Since $\hat{J}_{3}$ has discrete eigenvalues, normalisable eigenfunctions can be constructed. The general solution (in spherical polar variables $\mathbf{k} \rightarrow k, \theta, \varphi$ ) to

$$
\begin{aligned}
& \hat{J}_{3} \mathbf{v}_{m}(k, \theta, \varphi)=m \hbar \mathbf{v}_{m}(k, \theta, \varphi), \quad m=0, \pm 1, \pm 2, \ldots, \\
& \mathbf{k} \cdot \mathbf{v}_{m}(k, \theta, \varphi)=0,
\end{aligned}
$$

is a linear combination of

$$
\begin{aligned}
\boldsymbol{\alpha}_{m}(\theta, \varphi) & =e^{i(m-1) \varphi}\left(\begin{array}{c}
C \\
i C \\
-S e^{i \varphi}
\end{array}\right), \\
\boldsymbol{\beta}_{m}(\theta, \varphi) & =e^{i(m+1) \varphi}\left(\begin{array}{c}
i C \\
C \\
-i S e^{-i \varphi}
\end{array}\right), \\
C & =\cos \theta, S=\sin \theta
\end{aligned}
$$

with any functions of $k, \theta$ as coefficients (subject to $\mathbf{v}_{m}(k, \theta, \varphi)$ being singlevalued at $\left.\theta=0, \pi\right)$. The column vectors here are however not mutually orthogonal. Using the circular polarization basis vectors $\boldsymbol{\epsilon}^{( \pm)}(\hat{\mathbf{k}})$ of eq. (3.37), we can find an alternative construction. The orthonormal vectors $\boldsymbol{\epsilon}^{( \pm)}(\hat{\mathbf{k}})$ are

$$
\begin{gathered}
\boldsymbol{\epsilon}^{(+)}(\theta, \varphi)=\frac{e^{i \varphi}}{\sqrt{2}}\left(\begin{array}{c}
\cos \theta \cos \varphi-i \sin \varphi \\
\cos \theta \sin \varphi+i \cos \varphi \\
-\sin \theta
\end{array}\right), \\
\boldsymbol{\epsilon}^{(-)}(\theta, \varphi)=i \boldsymbol{\epsilon}^{(+)}(\theta, \varphi)^{*} .
\end{gathered}
$$

As expected, at $\theta=\pi$ we have $\varphi$-dependent limits:

$$
\begin{gathered}
\boldsymbol{\epsilon}^{(+)}(\pi, \varphi)=\frac{e^{2 i \varphi}}{\sqrt{2}}\left(\begin{array}{c}
-1 \\
i \\
0
\end{array}\right), \\
\boldsymbol{\epsilon}^{(-)}(\pi, \varphi)=-i \frac{e^{-2 i \varphi}}{\sqrt{2}}\left(\begin{array}{l}
1 \\
i \\
0
\end{array}\right) .
\end{gathered}
$$

We then find that any $\mathbf{v}_{m}(k, \theta, \varphi)$ obeying eq. (5.1) is a $(k, \theta)$ dependent linear combination of

$$
e^{i(m-1) \varphi} \boldsymbol{\epsilon}^{(+)}(\theta, \varphi), \quad e^{i(m+1) \varphi} \boldsymbol{\epsilon}^{(-)}(\theta, \varphi),
$$

subject again to being singlevalued at $\theta=0, \pi$.

The sets (5.2), (5.5) of $\hat{J}_{3}$ eigenfunctions are linearly related:

$$
\begin{aligned}
& \left(\begin{array}{l}
\boldsymbol{\alpha}_{m}(\theta, \varphi) \\
\boldsymbol{\beta}_{m}(\theta, \varphi)
\end{array}\right)=\frac{1}{\sqrt{2}}\left(\begin{array}{ll}
(1+C) & -i(1-C) \\
i(1-C) & (1+C)
\end{array}\right) \\
& \times\left(\begin{array}{cc}
e^{i(m-1) \varphi} & \boldsymbol{\epsilon}^{(+)}(\theta, \varphi) \\
e^{i(m+1) \varphi} & \boldsymbol{\epsilon}^{(-)}(\theta, \varphi)
\end{array}\right) ; \\
& \left(\begin{array}{cc}
e^{i(m-1) \varphi} & \boldsymbol{\epsilon}^{(+)}(\theta, \varphi) \\
e^{i(m+1) \varphi} & \boldsymbol{\epsilon}^{(-)}(\theta, \varphi)
\end{array}\right) \\
& =\frac{1}{2 \sqrt{2}}\left(\begin{array}{ll}
1+\sec \theta & i(\sec \theta-1) \\
-i(\sec \theta-1) & 1+\sec \theta
\end{array}\right)\left(\begin{array}{l}
\boldsymbol{\alpha}_{m}(\theta, \varphi) \\
\boldsymbol{\beta}_{m}(\theta, \varphi)
\end{array}\right) .
\end{aligned}
$$


Therefore, returning to eq. (5.1), we can write in general

$$
\begin{aligned}
\mathbf{v}_{m}(k, \theta, \varphi)= & a(k, \theta) e^{i(m-1) \varphi} \boldsymbol{\epsilon}^{(+)}(\theta, \varphi)+b(k, \theta) \\
& \times e^{i(m+1) \varphi} \boldsymbol{\epsilon}^{(-)}(\theta, \varphi),
\end{aligned}
$$

with $a(k, \theta), b(k, \theta)$ suitably behaved at $\theta=0, \pi$ but otherwise arbitrary. If $\mathbf{v}_{m^{\prime}}(k, \theta, \varphi)$ is another $\hat{J}_{3}$ eigenfunction for eigenvalue $m^{\prime} \hbar$ involving $a^{\prime}(k, \theta), b^{\prime}(k, \theta)$ we find:

$$
\begin{aligned}
& \left(\mathbf{v}_{m^{\prime}}, \mathbf{v}_{m}\right)=\int d^{3} k \mathbf{v}_{m^{\prime}}(k, \theta, \varphi)^{*} \cdot \mathbf{v}_{m}(k, \theta, \varphi) \\
= & 2 \pi \delta_{m^{\prime}, m} \int_{0}^{\infty} k^{2} d k \int_{0}^{\pi} \sin \theta d \theta\left(a^{\prime}(k, \theta)^{*} a(k, \theta)\right. \\
+ & \left.b^{\prime}(k, \theta)^{*} b(k, \theta)\right) .
\end{aligned}
$$

Thus, assuming $\mathbf{v}(k, \theta, \phi)$ is normalized, $\hat{k} \cdot \hat{\mathbf{S}}$ has a definite expectation value and a finite nonzero spread with respect to it. It may be emphasized that the two terms in eq. (5.7) are not related to eigenfunctions of $\hat{k} \cdot \hat{\mathbf{S}}$ (in $\mathcal{H}_{1}$ ) in any way, as these are not normalisable.

The general structure of $\hat{J}_{3}$ eigenfunctions in physical space, i.e., their azimuthal dependences, can be found using eq. (2.20). A transverse analytic signal $\mathbf{A}^{(+)}(x)$ obeying

$$
\hat{J}_{3} \mathbf{A}^{(+)}(x)=m \hbar \mathbf{A}^{(+)}(x)
$$

is a complex numerical linear combination of threecomponent amplitudes of the form (in spherical polar variables $\mathbf{x} \rightarrow r, \theta, \phi)$

$$
e^{i(m-1) \phi}\left(\begin{array}{c}
a(r, \theta, t) \\
i a(r, \theta, t) \\
c(r, \theta, t) e^{i \phi}
\end{array}\right), e^{i(m+1) \phi}\left(\begin{array}{c}
i b(r, \theta, t) \\
b(r, \theta, t) \\
c^{\prime}(r, \theta, t) e^{-i \phi}
\end{array}\right),
$$

subject to the wave equation for $\mathbf{A}^{(+)}(x)$, transversality, singlevaluedness and the positive frequency condition. In $\mathrm{x}$-space there is no analogue to the solutions of the form (5.5), since $\boldsymbol{\epsilon}^{( \pm)}(\hat{\mathbf{k}})$ are local in $\mathbf{k}$-space alone.

\section{Photon wave functions and vector potential in paraxial limit}

We begin with consequences of eqs. (3.2, 3.16):

$$
\begin{gathered}
\mathbf{v}(\mathbf{k}) \in \mathcal{M}: \quad \hat{a}_{j}(\mathbf{k})|\mathbf{v}\rangle=\frac{1}{\sqrt{\hbar}} v_{j}(\mathbf{k})|0\rangle, \\
\langle\mathbf{v}| \hat{a}_{j}(\mathbf{k})^{\dagger}=\frac{1}{\sqrt{\hbar}} v_{j}(\mathbf{k})^{*}\langle 0| .
\end{gathered}
$$

As discussed in Section 4, in the paraxial case it is the choice of $\mathbf{v}(\mathbf{k})$ that is suitably restricted, without affecting the operators $\hat{\mathbf{a}}(\mathbf{k}), \hat{\mathbf{a}}(\mathbf{k})^{\dagger}$.

The paraxial region in $\mathbf{k}$-space consists of wave vectors along and very close to the positive $z$-axis:

$$
\begin{aligned}
\mathbf{k}= & \left(\mathbf{k}_{\perp}, \sqrt{k^{2}-\mathbf{k}_{\perp}^{2}}\right)=k(\sin \theta \cos \varphi, \sin \theta \sin \varphi, \cos \theta) \\
& \simeq k\left(\theta \cos \varphi, \theta \sin \varphi, 1-\theta^{2} / 2\right), \quad 0 \leq \theta<<1,(5.12)
\end{aligned}
$$

where only terms upto quadratic in $\theta$ are retained in all relevant expressions. The condition on $\mathbf{v}(\mathbf{k})$ stated in eq. (4.18) is that it be negligible outside of the region (5.12):

$$
\mathbf{v}(\mathbf{k}) \approx 0 \text { unless } \theta<<1 \text {, i.e. }\left|\mathbf{k}_{\perp}\right|<<k .
$$

Then transversality gives

$$
v_{3}(\mathbf{k}) \simeq-\theta\left(\cos \varphi v_{1}(\mathbf{k})+\sin \varphi v_{2}(\mathbf{k})\right) .
$$

Qualitatively stated, $v_{3}(\mathbf{k})$ is one order of magnitude smaller than $v_{\perp}(\mathbf{k})$.

To apply these considerations to the exact $\hat{J}_{3}$ eigenfunctions $\mathbf{v}_{m}(k, \theta, \varphi)$ in eq. (5.7), we impose the paraxial property on $a(k, \theta), b(k, \theta)$ :

$$
a(k, \theta), b(k, \theta) \approx 0 \text { unless } \theta<<1 \text {. }
$$

We now see that if such a paraxial $\mathbf{v}_{m}(k, \theta, \varphi)$, with both $a(k, \theta)$ and $b(k, \theta)$ sharply peaked about $\theta=0$, is expanded in the total angular momentum eigenfunctions $\left\{\mathbf{Y}_{l, m}^{(a)}(\theta, \varphi)\right\}$ of eq. (3.24), on account of the uncertainty principle many terms with a large spread of $l$ values will be present. On the other hand, since the paraxial region (5.12) is preserved under rotations about the $z$-axis, individual $\hat{J}_{3}$ eigenfunctions remain useful in this regime; the label ' $m$ ' remains a 'good quantum number'. This explains the motivation to study $\mathbf{v}_{m}(k, \theta, \varphi)$ in this limit.

The vector potential in the paraxial limit shows some subtleties. We start with the analogues to the scalar equations (4.2, 4.3):

$$
\begin{aligned}
& \mathbf{A}^{(+)}(x)=\int_{0}^{\infty} d \omega \tilde{\mathbf{A}}^{(+)}(\mathbf{x} ; \omega) e^{-i \omega t} ; \\
& \left(\nabla^{2}+k^{2}\right) \tilde{\mathbf{A}}^{(+)}(\mathbf{x} ; \omega)=0, \quad \boldsymbol{\nabla} \cdot \tilde{\mathbf{A}}^{(+)}(\mathbf{x} ; \omega)=0 .
\end{aligned}
$$

When $\mathbf{v}(\mathbf{k})$ obeys eqs. (5.13, 5.14) there is some minimum $\omega_{\min }>0$; and consistent with eq. (4.19) we have:

$\tilde{\mathbf{A}}^{(+)}\left(\mathbf{x}_{\perp}, z ; \omega\right) \simeq \frac{e^{i k z}}{2 \pi \sqrt{\omega}} \int d^{2} \mathbf{k}_{\perp} \mathbf{v}\left(\mathbf{k}_{\perp}, k\right) e^{i \mathbf{k}_{\perp} \cdot \mathbf{x}_{\perp}-i \frac{\lambda}{2} \mathbf{k}_{\perp}^{2} z}$.

This obeys the PWE component wise, and the transversality condition:

$$
i \frac{\partial}{\partial z} \tilde{\mathbf{A}}^{(+)}\left(\mathbf{x}_{\perp}, z ; \omega\right)=\left(-k-\frac{\lambda}{2} \nabla_{\perp}^{2}\right) \tilde{\mathbf{A}}^{(+)}\left(\mathbf{x}_{\perp}, z ; \omega\right) ;
$$

$$
\nabla_{\perp} \cdot \tilde{\mathbf{A}}_{\perp}^{(+)}\left(\mathbf{x}_{\perp}, z ; \omega\right)+\frac{\partial}{\partial z} \tilde{\mathbf{A}}_{3}^{(+)}\left(\mathbf{x}_{\perp}, z ; \omega\right) \simeq 0 .
$$

In the PWE there is an extra term compared to eq. (4.8) as the factor $e^{i k z}$ has been retained; this in turn is because we wish to avoid extra terms in the transversality condition. Moreover this condition can be obeyed only approximately, to the same degree of accuracy as the paraxial condition. The subtleties involved in imposing transversality in the paraxial regime have been discussed in reference 23] This just reflects the fact that eq. (5.14) is an approximate statement. 


\section{Gaussian type examples}

To illustrate these ideas further, we consider the case where $\mathbf{v}(\mathbf{k})$ is proportional to a centred Gaussian factor characterized by a width parameter $w$ as in the scalar Laguerre-Gaussian case (4.20):

$$
\mathbf{v}(\mathbf{k})=\left(\begin{array}{c}
\mathbf{a}_{\perp}\left(\mathbf{k}_{\perp}\right) \\
c\left(\mathbf{k}_{\perp}\right)
\end{array}\right) e^{-w^{2} \mathbf{k}_{\perp}^{2} / 4}
$$

with $\mathbf{a}_{\perp}\left(\mathbf{k}_{\perp}\right), c\left(\mathbf{k}_{\perp}\right)$ polynomial in $\mathbf{k}_{\perp}$. Then the transversality condition (5.18b) reads

$$
\begin{array}{r}
\mathbf{k}_{\perp} \cdot \mathbf{a}_{\perp}\left(\mathbf{k}_{\perp}\right)+\left(k-\frac{\lambda}{2} \mathbf{k}_{\perp}^{2}\right) c\left(\mathbf{k}_{\perp}\right) \approx 0, \\
\text { i.e., } \quad\left(1-\frac{1}{2} \frac{\mathbf{k}_{\perp}^{2}}{k^{2}}\right) c\left(\mathbf{k}_{\perp}\right) \approx-\frac{\mathbf{k}_{\perp}}{k} \cdot \mathbf{a}_{\perp}\left(\mathbf{k}_{\perp}\right) .
\end{array}
$$

The structure of this condition shows again why in the paraxial regime we can impose transversality only approximately. For example, if we imagine the polynomials $\mathbf{a}_{\perp}\left(\mathbf{k}_{\perp}\right)$ to be preassigned, the expression $\left(k^{2}-\frac{1}{2} \mathbf{k}_{\perp}^{2}\right)$ will almost certainly not be a factor in them, so that $c\left(\mathbf{k}_{\perp}\right)$ cannot be a polynomial if eq. (5.20) is demanded as an exact quality. To leading paraxial order, then, we have

$$
c\left(\mathbf{k}_{\perp}\right) \simeq-\left(1+\frac{1}{2} \frac{\mathbf{k}_{\perp}^{2}}{k^{2}}\right) \frac{\mathbf{k}_{\perp}}{k} \cdot \mathbf{a}_{\perp}\left(\mathbf{k}_{\perp}\right),
$$

and as in eq. (5.14) $c\left(\mathbf{k}_{\perp}\right)$ is one order of magnitude smaller than $\mathbf{a}_{\perp}\left(\mathbf{k}_{\perp}\right)$.

The scalar Laguerre-Gauss modes of eq. (4.20) can be extended to define corresponding vectorial photon modes in two ways, with the two structures in eq. (5.2). Choose an eigenvalue $m \hbar$ for $\hat{J}_{3}$, and an index $p=0,1,2, \ldots$. Then upto an overall numerical constant, we can take $\mathbf{v}(\mathbf{k})$ to be (in cylindrical coordinates $\mathbf{k}_{\perp} \rightarrow(\rho, \varphi)$ ):

$$
\begin{aligned}
& \mathbf{v}_{m, p}\left(\mathbf{k}_{\perp}\right)=e^{i(m-1) \varphi}\left(\begin{array}{c}
a(\rho) \\
i a(\rho) \\
c(\rho) e^{i \varphi}
\end{array}\right), \\
& a(\rho)=w\left(\frac{i w \rho}{\sqrt{2}}\right)^{|m-1|} L_{p}^{|m-1|}\left(w^{2} \rho^{2} / 2\right) e^{-w^{2} \rho^{2} / 4}, \\
& c(\rho) \simeq-\left(1+\frac{\rho^{2}}{2 k^{2}}\right) \frac{\rho}{k} a(\rho) .
\end{aligned}
$$

This corresponds to the $\boldsymbol{\alpha}_{m}$ structure in eq. (5.2). The other possibility, with the structure of $\boldsymbol{\beta}_{m}$ in eq. (5.2), is:

$$
\begin{aligned}
& \mathbf{v}_{m, p}^{\prime}\left(\mathbf{k}_{\perp}\right)=e^{i(m+1) \varphi}\left(\begin{array}{c}
i a^{\prime}(\rho) \\
a^{\prime}(\rho) \\
c^{\prime}(\rho) e^{-i \varphi}
\end{array}\right), \\
& a^{\prime}(\rho)=w\left(\frac{i w \rho}{\sqrt{2}}\right)^{|m+1|} L_{p}^{|m+1|}\left(w^{2} \rho^{2} / 2\right) e^{-w^{2} \rho^{2} / 4} \\
& c^{\prime}(\rho) \simeq-i\left(1+\frac{\rho^{2}}{2 k^{2}}\right) \frac{\rho}{k} a^{\prime}(\rho) .
\end{aligned}
$$

( The primes here do not mean derivatives with respect to the argument)

\section{General $\hat{J}_{3}$ eigenfunctions in paraxial regime}

Finally we consider the behavior of the general $\hat{J}_{3}$ eigenfunction (5.7) in the paraxial limit. With the condition (5.12) this takes the approximate form

$$
\begin{gathered}
\mathbf{v}_{m}(k, \theta, \varphi) \simeq \frac{1}{\sqrt{2}} \cdot e^{i(m-1) \varphi} a(k, \theta)\left(\begin{array}{c}
1-\frac{\theta^{2}}{2} e^{i \varphi} \cos \varphi \\
i-\frac{\theta^{2}}{2} e^{i \varphi} \sin \varphi \\
-\theta e^{i \varphi}
\end{array}\right) \\
+\frac{i}{\sqrt{2}} \cdot e^{i(m+1) \varphi} b(k, \theta)\left(\begin{array}{c}
1-\frac{\theta^{2}}{2} e^{-i \varphi} \cos \varphi \\
-i-\frac{\theta^{2}}{2} e^{-i \varphi} \sin \varphi \\
-\theta e^{-i \varphi}
\end{array}\right),
\end{gathered}
$$

consistent with eq. (5.14). From eq. (3.36) we also have, in this limit,

$$
\hat{\mathrm{S}}_{3} \boldsymbol{\epsilon}^{( \pm)}(\hat{\mathbf{k}}) \simeq \pm \hbar\left(1-\theta^{2} / 2\right) \boldsymbol{\epsilon}^{( \pm)}(\hat{\mathbf{k}}),
$$

for the two terms in eq. (5.24). (However, as noted after eq. (3.36), these terms are not eigenfunctions of $\hat{\mathbf{k}} \cdot \hat{\mathbf{S}}$ in $\left.\mathcal{H}_{1}\right)$.

We see that to order $\theta$ (but not to order $\left.\theta^{2}\right)$ the $a(k, \theta)$ term $(b(k, \theta)$ term) in eq. (5.24) has 'spin' angular momentum along the $z$-axis of amount $\hbar(-\hbar)$, (right/left circular polarization), so it has 'orbital' angular momentum along the $z$-axis of amount $(m-1) \hbar((m+1) \hbar)$. But these are approximate statements. Apart from being valid only to order $\theta$, there are no consistent definitions of 'orbital' and 'spin' angular momenta for classical light or for photons.

\section{SUMMARY AND CONCLUSIONS}

In this work we have tried to give a comprehensive treatment, starting from first principles, of the angular momentum of electromagnetic radiation in both classical and quantum domains, and covering both general and paraxial situations. The free Maxwell field equations are invariant under the action of the ten parameter Poincaré group which leads to the ten basic conservation laws or COM's. We have developed several ways of expressing them and in particular in terms of analytic signals making the transition to the quantum theory very easy.

The use of the classical Hilbert space $\mathcal{M}$ eq. (2.31), made up of suitable solutions of the classical Maxwell equations, serving in the quantum case as the set of all single photon wave functions is a salient feature of our treatment. For single photons every component $\hat{J}_{j}$ of the total angular momentum is in principle a physical observable, not just the component $\hat{\mathbf{p}} \cdot \hat{\mathbf{J}}$ along the momentum direction as is sometimes stated. This is made particularly clear by the properties of the basis of total angular 
momentum eigenfunctions $\left\{\mathbf{Y}_{l m}^{(a)}(\hat{\mathbf{k}})\right\}$ for photons, to be contrasted with the plane wave energy momentum eigenfunctions.

The particular focus here has been on the extensively discussed separation of the total angular momentum of light into 'orbital' and 'spin' parts, reminiscent in the definition of the case of massive particles. We have paid special attention to their definitions as dynamical variables, and in the quantum theory to the unitary transformations they generate on single photon wave functions. As has been recognized for some time, neither of them has the algebraic properties - CR's - characteristic of angular momentum in quantum mechanics. This in turn is related to the well-known result of Newton and Wigner that the concept of position with reasonable properties is undefined for photons - if it were, then so would orbital angular momentum in the sense of quantum mechanics, and then spin. In actual fact, the total angular momentum and the proposed 'spin' vector together realize the Lie algebra of the Euclidean group E(3), though all of them have dimensions of action. From the theory of the UIR's of this group it is well known that the eigenvectors of the 'translation generators' $\hat{\mathbf{S}}$ are 'ideal vectors' obeying Dirac delta function normalization over the sphere $\mathbb{S}^{2}$. It follows that normalisable eigenvectors of $\hat{J}_{3}$, constructed explicitly in Section V, are never expressible as discrete linear combinations of $\hat{S}_{3}$ 'eigenvectors'. As can be easily verified, in any single photon state $\mathbf{v}_{m}(k, \theta, \varphi)$ in eq. (5.7), $\hat{S}_{3}$ has an expectation value in the interval $(-\hbar, \hbar)$ and a non vanishing variance or spread $\left(\Delta \hat{S}_{3}\right)^{2}$.

The transversality of the free Maxwell field, both of the field strengths and of the vector potential in the radiation gauge, is a requirement that has a profound influence on the quantization process as well as on the description of single photon states. We have kept careful track of this in the process of quantization and in the definition and passage to the paraxial regime. It is to be appreciated that any choice of a transverse polarization basis $\left\{\boldsymbol{\epsilon}_{\alpha}(\mathbf{k})\right\}$ in wave vector space brings with it a certain degree of phase freedoms or phase ambiguities, and to this degree the action of the total angular momentum operator $\hat{J}_{3}$ on single photon wave functions will also be affected. For this reason we have avoided as far as possible any choice of such bases. We have examined how and in what sequence these two processes should be implemented, maintaining consistency from both physical and mathematical points of view. We have tried to present convincing arguments to show that in the quantum case the paraxial limit should be taken after, not before, quantization.

Our treatment of the paraxial limit has been to the lowest nontrivial order. We have emphasized that most solutions of the paraxial wave equation are in fact not paraxial; to be paraxial definite restrictions have to be imposed on a general solution. This as we have seen has consequences described in the previous paragraph. Even to this order, we have seen that the transversality condition makes physical sense only as an approximate, and not as an exact, condition. In this way, an appropriate extension of the much used Laguerre-Gaussian modes from the scalar to the vector case has been derived.

From the point of view of special relativity, the appropriate framework for setting up a systematic procedure to handle higher order paraxial approximations is the front form of relativistic dynamics. This is one of three forms elaborated by Dirac long ago [36]. This method has been used in the past to handle the polarization of light consistently to leading paraxial order. A treatment of higher orders by this method, both classically and in quantum theory, will be presented elsewhere.

In this work we have emphasized the description and properties of single photon states, and exploited the fact that the manifold of solutions of the classical Maxwell equations goes over naturally to the space of single photon wave functions. The treatment of two or more multiphoton states will then follow by using standard quantum mechanical methods subject to the requirements of Bose statistics. This and other related aspects will be taken up elsewhere. It is our hope that the formalism developed here will be found useful by those working in the field and in particular to understand the properties of interesting quantum situations involving non-classical and entangled light with OAM. With our improved understanding of the algebraic properties of $\hat{\mathbf{L}}$ and $\hat{S}$ in particular that they are not angular momenta, it seems preferable to expand the OAM to 'Optical Angular Momentum'.

\section{Acknowledgements}

Arvind acknowledges funding from DST India under Grant No. EMR/2014/000297. NM thanks the Indian National Science Academy for enabling this work through the INSA Distinguished Professorship.
[1] D. L. Andrews and M. Babiker, eds., The Angular Momentum of Light (Cambridge University Press, 2012).

[2] E. Wolf, Introduction to the Theory of Coherence and Polarization of Light (Cambridge University Press, 2007), ISBN 978-0-521-82211-4.

[3] D.-X. Chen, P. Zhang, R.-F. Liu, H.-R. Li, H. Gao, and F.-L. Li, Physics Letters A 379, $2530 \quad$ (2015), ISSN 0375-9601, URL
http://www.sciencedirect.com/science/article/pii/S037596011

[4] G. Kulkarni, R. Sahu, O. S. Magaa-Loaiza, R. W. Boyd, and A. K. Jha, Nature Communications 8, 1054 (2017), URL https://doi .org/10.1038/s41467-017-01215-x.

[5] X. Zou and W. Mathis, Phys. Rev. A $71,042324 \quad$ (2005), URL https://link.aps.org/doi/10.1103/PhysRevA.71.042324.

[6] F. Bouchard, R. Fickler, R. W. Boyd, and 
E. Karimi, in Frontiers in Optics 2016 (Optical Society of America, 2016), p. FF5D.4, URL http://www . osapublishing. org/abstract . cfm?URI=FiO-20126H

[7] Z.-K. Su, F.-Q. Wang, R.-B. Jin, R.-S. Liang, and S.-H. Liu, Optics Communications 281, 5063 (2008), ISSN 0030-4018, URL http://www.sciencedirect.com/science/article/pii/sc

[8] L.-P. Deng, H. Wang, and K. Wang, J. Opt. Soc. Am. B 24, 2517 (2007), URL http://josab.osa.org/abstract.cfm?URI=josab-24-9-2517

[9] J. C. Garca-Escartn and P. Chamorro-Posada, Journal of Optics 13, $064022 \quad$ (2011), URL http://stacks.iop.org/2040-8986/13/i=6/a=064022

[10] C. Perumangatt, N. Lal, A. Anwar, S. G. Reddy, and R. Singh, Physics Letters A 381, 1858 (2017), ISSN 0375-9601, URL http://www.sciencedirect.com/science/article/pii/sc

[11] F. M. Spedalieri, Optics Communications 260, $340 \quad$ (2006), ISSN 0030-4018, URL http://www.sciencedirect.com/science/article/pii/s

[12] M. Mafu, A. Dudley, S. Goyal, D. Giovannini, M. McLaren, M. J. Padgett, T. Konrad, F. Petruccione, N. Lütkenhaus, and A. Forbes, Phys. Rev. A 88, 032305 (2013), URL https://link.aps.org/doi/10.1103/PhysRevA.88.032305

[13] H. H. Arnaut and G. A. Barbosa, Phys. Rev. Lett. 85, $286 \quad$ (2000), URL https://link.aps.org/doi/10.1103/PhysRevLett.85.286

[14] A. Mair, A. Vaziri, G. Weihs, and A. Zeilinger, Nature 412, 313 (2001), URL https://www.scopus. com/inward/record.uri?eid=2-s2.0

[15] P. Zhang, X.-F. Ren, X.-B. Zou, B.-H. Liu, Y.-F. Huang, and G.-C. Guo, Phys. Rev. A 75, 052310 (2007), URL https://link.aps.org/doi/10.1103/PhysRevA.75.052310

[16] H. Larocque, J. Gagnon-Bischoff, D. Mortimer, Y. Zhang, F. Bouchard, J. Upham, V. Grillo, R. W. Boyd, and E. Karimi, Opt. Express 25, $19832 \quad$ (2017), URL http://www opticsexpress.org/abstract.cfm?URI=0e-25-17-18RaRematical Physics 17] M. Lassen, G. Leuchs, and U. Andersen, https://ww scopus com/inward/record uri?eid=2-s2.0

[18] A. Marino, V. Boyer, R. Pooser, P. Lett, K. Lemons, and K. Jones, Physical Review Letters 101 (2008), URL https://www. scopus.com/inward/record.uri?eid=2-s2.0-50664-1

[19] J. Leach, B. Jack, J. Romero, A. Jha, A. Yao, S. Franke-Arnold, D. Ireland, R. Boyd, S. Barnett, and M. Padgett, Science 329, 662 (2010), URL https : //www . scopus . com/inward/record.uri?eid=2-s2 .0

[20] J. Leach, M. Padgett, S. Barnett, S. FrankeArnold, and J. Courtial, Physical Re- view Letters $\mathbf{8 8}, 2579011 \quad$ (2002), URL https://www.scopus. com/inward/record.uri?eid=2-s2.0-0037166 FE5DA4en, M. W. Beijersbergen, R. J. C. Spreeuw, and J. P. Woerdman, Phys. Rev. A 45, 8185 (1992), URL https://link.aps.org/doi/10.1103/PhysRevA.45.8185

[22] G. F. Calvo, A. Picón, and E. Bagan, 0P\$g80061BQ v. A $\mathbf{7 3}, 013805 \quad$ (2006), URL https://link.aps.org/doi/10.1103/PhysRevA.73.013805

[23] I. H. Deutsch and J. C. Garrison, Phys. Rev. A 43, 2498 (1991), URL https://link.aps.org/doi/10.1103/PhysRevA.43.2498

[24] E. Wigner, Annals of Mathematics 40, 149 (1939), ISSN 0003486X, URL http://www.jstor.org/stable/1968551.

[25] T. D. Newton and E. P. Wigner, Rev. Mod. Phys. 21, 400 (1949), URL

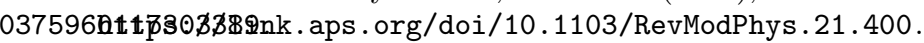
[26] R. Nityananda and S. Sridhar, Annals of Physics 341, 117 (2014), ISSN 0003-4916, URL 00304Ch805010 [27] N. Mukunda, S. Chaturvedi, and R. Simon, J. Opt. Soc. Am. A 31, 1141 (2014), URL http://josaa.osa.org/abstract.cfm?URI=josaa-31-6-1141

[28] Arvind, S. Chaturvedi, and N. Mukunda, Physics Letters A 381, 3005 (2017), ISSN 0375-9601, URL http://www.sciencedirect.com/science/article/pii/S037596011

[29] E. W. Max Born, Principles of optics (Cambridge University Press, 1997), 6th ed., ISBN 0-521-63921-2.

[30] S. J. van Enk and G. Nienhuis, EPL (Europhysics Letters) $\mathbf{2 5}, 497 \quad$ (1994), URL

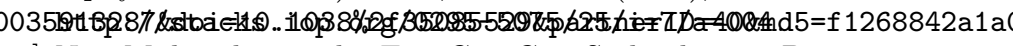

[31] N. Mukunda and E. C. G. Sudarshan, Pramana 27, 1 (1986), ISSN 0973-7111, URL https://doi.org/10.1007/BF02846324.

[32] V. F. W. a. John M. Blatt, Theoretical Nuclear Physics (Springer-Verlag New York, 1979), 1st ed., ISBN 978-14612-9961-5,978-1-4612-9959-2.

[33] A. J. Devaney and E. Wolf, Journal of https://doi.org/10.1063/1.1666629, https://doi.org/10.1063/1.1666629

URL

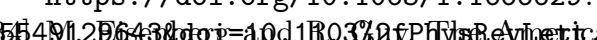

2Ma6l3602kpart tner ID $=40$ \&n Monthly 86, 571 (1979), ISSN 00029890, 19300972, URL http://www.jstor.org/stable/2320587.

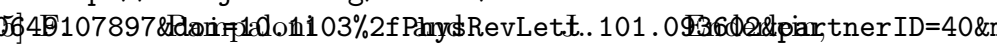
arXiv:physics/0410021 (2004), URL

https://arxiv.org/abs/physics/0410021.

[36] P. A. M. Dirac, Rev. Mod. Phys. 21, 392 (1949), URL 